\title{
Impact of Numerical Relativity information on effective-one-body waveform models
}

\author{
Alessandro Nagar, ${ }^{1,2,3}$ Gunnar Riemenschneider, ${ }^{4,5}$ and Geraint Pratten ${ }^{6}$ \\ ${ }^{1}$ Centro Fermi - Museo Storico della Fisica e Centro Studi e Ricerche "Enrico Fermi", Rome, Italy \\ ${ }^{2}$ INFN Sezione di Torino, Via P. Giuria 1, 10125 Torino, Italy \\ ${ }^{3}$ Institut des Hautes Etudes Scientifiques, 91440 Bures-sur-Yvette, France \\ ${ }^{4}$ Dipartimento di Fisica, Università di Torino, via P. Giuria 1, I-10125 Torino, Italy \\ ${ }^{5}$ Physik-Department, Technische Universität München, \\ Fames-Franck-Straße 1, 85748 Garching, Germany \\ ${ }^{6}$ Universitat de les Illes Balears, IAC3-IEEC, 07122 Palma de Mallorca, Spain
}

(Dated: March 21, 2017)

\begin{abstract}
We present a comprehensive comparison of the spin-aligned effective-one-body (EOB) waveform model of Nagar et al. [Phys. Rev. D 93, 044046 (2016)], informed using 40 numerical-relativity (NR) datasets, against a set of $149 \ell=m=2 \mathrm{NR}$ waveforms freely available through the Simulation Extreme Spacetime (SXS) catalog. We find that, without further calibration, these EOBNR waveforms have unfaithfulness - at design Advanced-LIGO sensitivity and evaluated with total mass $M$ varying as $10 M_{\odot} \leq M \leq 200 M_{\odot}$-always below $1 \%$ against all $\mathrm{NR}$ waveforms except for three outliers, that still never exceed the $3 \%$ level; with a minimal retuning of the (effective) next-to-next-to-next-toleading-order spin-orbit coupling parameter for the non-equal-mass and non-equal-spin sector, that only needs three more NR waveforms, one is left with another two (though different) outliers, with maximal unfaithfulness of up to only $2 \%$ for a total mass of $200 M_{\odot}$. We show this is the effect of slight inaccuracies in the phenomenological description of the postmerger waveform of Del Pozzo and Nagar arXiv:1606.03952 that was constructed by interpolating over only 40 NR simulations. We argue that this is easily fixed by using either an alternative ringdown description (e.g., the superposition of quasi-normal-modes) or an improved version of the phenomenological representation. By analyzing a NR waveform with mass ratio 8 and dimensionless spins +0.85 obtained with the BAM code, we conclude that the model would benefit from NR simulations specifically targeted at improving the postmerger-ringdown phenomenological fits for mass ratios $\gtrsim 8$ and spins $\gtrsim 0.8$. We finally show that some of the longest SXS $q=7$ waveforms suffer from systematic uncertainties in the postmerger-ringdown part that are interpreted as due to unphysical drifts of the center of mass: thus some care should be applied when these waveforms are used for informing analytical models.
\end{abstract}

PACS numbers: $04.30 . \mathrm{Db}, 04.25 . \mathrm{Nx}, 95.30 . \mathrm{Sf}$,

\section{INTRODUCTION}

Effective-one-body (EOB) [1-4] waveforms informed by numerical relativity (NR) simulations [5] 7] have played a central role in the detection, subsequent parameter-estimation [8] analyses and GR tests [9] of the gravitational-wave (GW) observations GW150914 10 and GW151226 [11, 12 announced in 2015. EOB waveforms have also been employed to build frequencydomain, phenomenological models for the inspiral, merger and ringdown stages of the $\mathrm{BBH}$ coalescence [13]. Those models were also used to infer the properties 8 and carry out tests 9 of GR with GW150914 and GW151226. There are currently two avatars of the EOBNR models: the SEOBNRv* lineage, that was used both in its SEOBNRv2 (spin-aligned, nonprecessing) and SEOBNRv3 (generic, precessing, spins) [14, 15] realizations to analyze LIGO/Virgo data [16. On the other hand, there is the SEOBNR_ihes avatar [17, that is currently restricted to aligned-spins (see however Ref. 18 for its extension to generically oriented spins) and has not yet been used in the analysis of experimental data. Though both models embody the same conceptual idea (i.e., the two body dynamics is represented as the dynamics of an effective particle whose conservative part is driven by some effective potential), there are several nontrivial differences. Let us just briefly quote some: (i) the choice of the resummation of the potential of the nonspinning sector; (ii) the treatment of spin-spin interaction; (iii) the incorporation of the (spinning) testparticle limit; (iv) the spin-gauge used for the spin-orbit sector as well as the approach to resumming the gyrogravitomagnetic functions; (v) the structure of the nextto-quasi-circular (NQC) corrections; (vi) the resummation of the residual waveform phases $\delta_{\ell m}$; (vii) the setup of low-eccentricity initial data; (viii) the choice of parameters that are informed through NR simulations. The SEOBNRv2 model 19 employed in the LIGO/Virgo data analyses has recently been upgraded to its SEOBNRv4 realization 20. This new model features several analytical upgrades with respect to SEOBNRv2, including: (i) the use of the full $4 \mathrm{PN}$ EOB interaction potential, as obtained in Ref. 21] (and notably already incorporated in the SEOBNR_ihes model 17]) and (ii) the next-to-next-to-leading order correction to the $(2,2)$ residual amplitud $\Phi^{1}$ correction and phase of the factor-

\footnotetext{
1 The performance of this term, obtained from the results of 22 was already discussed in Ref. [23], where it was argued that ad-
} 
ized waveform [6, 25, 27] that have been recasted from recent $\mathrm{PN}$ results 22 . In addition, the model is visibly improved with respect to SEOBNRv2 by calibrating it to a set of $141 \mathrm{NR}$ waveforms, instead of the original $38 \mathrm{NR}$ waveforms that were available in the calibration of SEOBNRv2. This procedure allows the new EOBNR waveforms to have faithfulness (at Advanced-LIGO design sensitivity) above $99 \%$ against all the NR waveforms discussed in Ref. 20, including 16 additional waveforms used for validation, when maximizing only on the initial phase and time.

Reference [17, hereafter Paper I, discussed the performances of the SEOBNR_ihes model. Although being analytically different from SEOBNRv2, that model was similarly improved by using the same $38 \mathrm{NR}$ waveforms used for the calibration of SEOBNRv2 in Ref. [19]. Now that most of the 141 waveforms used for the NR calibration of SEOBNRv4 in [20] are publicly available through the SXS catalog [28, it is urgent to test the robustness of the EOBNR model of Paper I on such an extended sample of configurations. Doing this check is the main aim of this paper. We shall show that the model of Paper I, once interpolated to arbitrary mass-ratios and spins, will provide a dominant $\ell=m=2$ waveform mode whose unfaithfulness is always below $3 \%$ against all available $\mathrm{NR}$ waveforms. More precisely, we will see that it is always below (or equal to) $1 \%$ except for two outliers that are slightly above this threshold. We then proceed to add four more waveforms to the 39 used in Paper I to slightly retune the model in order to provide a slightly more reliable (effective) representation of the spin-orbit interaction (as well as of the postmerger-ringdown in a few cases) that yields values of the unfaithfulness always below $1 \%$.

The paper is organized as follows. In Sec. II we briefly summarize the the NR data used in this paper. The cross-check of the SEOBNR model of Paper I against the spin-aligned waveforms publicly available in the SXS catalog is done in Sec. III. The improvement of the model, that is needed for just a few SXS NR waveforms, is discussed in Sec. IV] where it is found that the largest source of inaccuracy comes from the phenomenological description of the postmerger-ringdown phase based on the interpolating fit of Ref. 29]. Section V] discusses in detail the EOB/NR comparisons for $q=7$, notably pointing out systematic effects in the SXS waveforms due to the unphysical drift of the center of mass of the system. Finally, Sec. VI, explores the uncertainties of the model at the boundary of the "information" domain, by performing explicit comparisons with an equal-spin waveform with mass ratio $q \equiv m_{1} / m_{2}=8$ and dimensionless

ditional resummation procedures might be needed to improve the behavior of the waveform amplitude towards merger. We recall that additional resummations (through Padé approximants) were also found useful to be applied to the residual factorized correction to the waveform phase [24]. spins $\chi_{1}=\chi_{2}=+0.85$ obtained using the BAM code. We argue that the largest source of uncertainty comes from the imperfect modeling of the postmerger-ringdown phase. This is the part of the model that needs the most radical improvements. After the conclusions in Sec. VII, the paper is complemented by an Appendix that summarizes some technical material that is needed in the main text.

\section{NUMERICAL RELATIVITY DATA}

The numerical waveforms are taken from two independent sets: the publicly available SXS catalog 28] produced with the Spectral Einstein Code (SpEC) [11, 3043. as well as a single waveform produced by the BAM code 4446 .

We use 149 waveforms from the public SXS catalog [47] to validate the model. Conservative error bars are estimated by computing the phase difference at the NR merger (defined as the peak of the $\ell=m=2$ waveform amplitude) of the highest-resolution $(\mathrm{H})$ waveform between the highest and second highest $(\mathrm{SH})$ resolutions

$$
\left.\delta \phi_{\mathrm{mrg}}^{\mathrm{NR}} \equiv\left(\phi^{\mathrm{H}}(t)-\phi^{\mathrm{SH}}(t)\right)\right|_{t=t_{\mathrm{mrg}}^{\mathrm{H}}} .
$$

This quantity is monotonically increasing up to merger and is zero at the start of the two simulations. For those simulations for which we only have a single resolution available, no error bar is given. For the SXS simulations, we use the asymptotic waveforms that have been extrapolated to future null infinity. As in Paper I, we use the $N=3$ extrapolation order for the highest available resolutions in each simulation. The complete information for the NR runs used in this paper (and in particular the uncertainties at merger) can be found in Tables $\mathrm{V}$. IX.

In addition to the SXS simulations, we also use a BAM $(8,+0.85,+0.85)$ waveform corresponding to approximately 15.7 gravitational wave cycles before merger [48].

\section{CHECKING THE SEOBNR_IHES MODEL OF PAPER I}

\section{A. Summary of the available analytical flexibility}

The SEOBNR_ihes model NR informed in Paper I has the following analytical flexibility: (i) an effective $5 \mathrm{PN}$ coefficient $a_{6}^{c}$ entering the nonspinning sector of the model; (ii) an effective next-to-next-to-next-to-leadingorder (NNNLO) spin-orbit parameter $c_{3}$, that is tuned in order to make the spin-orbit interaction stronger or milder with respect to the simple NNLO (resummed) analytical prediction encoded in the gyro-gravitomagnetic functions $G_{S}$ and $G_{S_{*}}$ [49 51 , see Eqs. (41) and (42) of [51]; (iii) a parameter $\Delta t_{\mathrm{NQC}}$ aimed at connecting the EOB and NR time axis, that is given by Eq. (21) 
of Paper I; (iv) four next-to-quasi-circular (NQC) parameters $\left(a_{1}, a_{2}, b_{1}, b_{2}\right)$ entering the $\ell=m=2$ waveform $h_{22} \equiv h_{22}^{\text {circ }} \hat{h}_{22}^{\mathrm{NQC}}\left(a_{1}, a_{2}, b_{1}, b_{2}\right)$ (see Eq. (95) of [51]) that are determined by imposing osculation between the EOB and NR amplitude and frequency (and their time derivatives) close to merger 2 ? (v) the phenomenological description of the postmerger-ringdown waveform part introduced in Ref. [52. In Paper I, in order to accomplish a very accurate description of both the NQC corrections and the ringdown, the exact values of the NR NQC-extraction point was used to determine the parameters $\left(a_{i}, b_{i}\right)$, as well as the primary ringdown fit of [52], instead of some effective interpolation based on a sparse number of simulations. This choice (that is behind the central result of Paper I, the computation of the unfaithfulness in Fig. 9 and Fig. 21) was done in order to reduce the systematics in the determination of $c_{3}$ ( or $a_{6}^{c}$ ) related to the small sample of NR waveform data available, with rather little information available outside the equal-mass, equal-spin regime. By contrast, here we start by using the same values of $\left(a_{6}^{c}, c_{3}\right)$ determined in Paper $\mathrm{I}$, but we follow a different route to obtain the NQC parameters and to model the postmerger-ringdown waveform. More precisely: (i) the postmerger-ringdown waveform description adopted here is given by the global interpolating fits of Ref. 29] for the spin-aligned case and a special fit (presented here for the first time) for the nonspinning case (see Sec. III B below); (ii) we similarly use a different treatment for the NQC corrections: for the nonspinning case, the NQC parameters $\left(a_{i}, b_{i}\right)$ are obtained as fits of the "exact" coefficients determined after iterations (because then $\hat{h}_{22}^{\mathrm{NQC}}$ is included in the radiation sector [51]) by imposing osculation with the actual NR waveform data point; on the contrary, for the spinning case, such fits are not sufficiently accurate so we instead present two different fits to the NR NQC-extraction point with the final NQC parameters being obtained after several iterations.

\section{B. The nonspinning sector}

We use the functional form of $a_{6}^{c}(\nu)$ obtained in Paper I, that is $a_{6}^{c}(\nu)=3097.3 \nu^{2}-1330.6 \nu+81.38$ where $\nu \equiv m_{1} m_{2} / M^{2}$ is the symmetric mass ratio. This fit was determined using the first nine datasets in Table $\mathrm{V}$, with mass ratios $1 \leq q \leq 9.99$, plus a $q=1$ 15-orbit long dataset mentioned in Table I of Paper I. Here and below (as it was the case of Paper I) we use waveforms that have been extrapolated to future null infinity by using a polynomial of order $N=3$, using the highest resolution available in the SXS catalog. In addition, for each

\footnotetext{
2 More precisely, the NR waveform point used to do so is located at $t_{\mathrm{NQC}}^{\mathrm{NR}} / M=\left(t_{\mathrm{mrg}}^{\mathrm{NR}}+2\right) / M$, where $t_{\mathrm{mrg}}^{\mathrm{NR}}$ is the NR merger time defined as usual as the peak of the $\ell=m=2$ waveform amplitude and $M \equiv m_{1}+m_{2}$ the total mass of the system.
}

of these datasets one can determine (after a few iterations) the corresponding NQC parameters $\left(a_{1}, a_{2}, b_{1}, b_{2}\right)$ that parametrize the $\ell=m=2$ NQC factor as

$$
\hat{h}_{22}^{\mathrm{NQC}}=\left(1+a_{1} n_{1}+a_{2} n_{2}\right) e^{\mathrm{i}\left(b_{1} n_{1}^{\prime}+b_{2} n_{2}^{\prime}\right)},
$$

where $\left(n_{1}, n_{2}, n_{1}^{\prime}, n_{2}^{\prime}\right)$ are functions that explicitly depend on the radial momentum and that are listed in Eq. (96) of Ref. 51. The obtained values $\left(a_{i}, b_{i}\right)$ can then be accurately fit with a rational function in terms of the variable $X_{\nu} \equiv 1-4 \nu$. Using this procedure, we obtain

$$
\begin{aligned}
a_{1} & =-0.08052 \frac{1-2.0033 X_{\nu}^{2}}{1+3.0859 X_{\nu}^{2}}, \\
a_{2} & =1.52995 \frac{1+1.1643 X_{\nu}^{2}}{1+1.9203 X_{\nu}^{2}} \\
b_{1} & =0.146768\left(0.0742 X_{\nu}+1.01691256\right), \\
b_{2} & =0.896911\left(-0.6107 X_{\nu}+0.94295129\right) .
\end{aligned}
$$

For the phenomenological description of the postmergerringdown part, we (i) first produce the postmerger primary fits as in [52] and then (ii) fit the coefficients as functions of $\nu$. Referring the reader to Eqs. (4)-(10) of Ref. [52 for the coefficients entering the fit, we obtain the following $\nu$ dependences:

$$
\begin{aligned}
\alpha_{21}(\nu) & =-0.3396 \nu^{2}+0.0165 \nu+0.1817, \\
\alpha_{1}(\nu) & =-0.1809 \nu^{2}+0.0216 \nu+0.0872, \\
c_{3}^{A}(\nu) & =0.9211 \nu-0.58477 \\
c_{3}^{\phi}(\nu) & =-2.225 \nu+4.612 \\
c_{4}^{\phi}(\nu) & =-7.2958 \nu+3.9702 \\
\Delta \omega(\nu) & =1.13387 \nu^{2}-0.006827 \nu+0.114279, \\
A_{22}^{\operatorname{mrg}}(\nu) & =1.232 \nu^{2}+0.3113 \nu+1.4208 .
\end{aligned}
$$

Note that in order to reduce systematic uncertainties, the primary fit behind these numbers have been obtained by using the values of the final black hole mass and angular momentum $\left(M_{f}, j_{f}\right)$ as given in the metadata. txt file for each SXS simulation. Evidently, when interpolating all over the parameter space we use $\left(M_{f}, j_{f}\right)$ as provided by the NR fits of Healy et al. [53]. By contrast, the QNMs frequencies are not computed by using the interpolating fits of Ref. 54 but are directly interpolated (with a cubic spline interpolant) from the the tables provided on E. Berti's website [55].

Since in Paper I we were not using interpolating fits for either the NQC parameters or the postmerger-ringdown ones, it is interesting to evaluate again the quality of the nonspinning model in terms of unfaithfulness. The EOB/NR unfaithfulness (as function of the total mass $M$ of the binary) is defined as

$$
\bar{F}(M) \equiv 1-\max _{t_{0}, \phi 0} \frac{\left\langle h_{22}^{\mathrm{EOB}}, h_{22}^{\mathrm{NR}}\right\rangle}{\left\|h_{22}^{\mathrm{EOB}}||\right\| h_{22}^{\mathrm{NR}} \|},
$$

where $\left(t_{0}, \phi_{0}\right)$ are the initial time and phase, $\|h\| \equiv$ $\sqrt{\langle h, h\rangle}$, and the inner product between two waveforms 


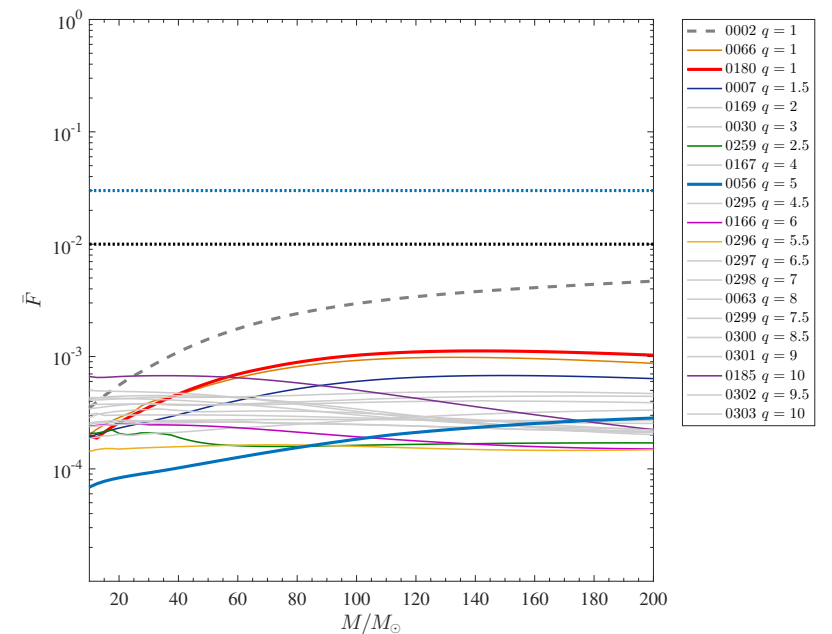

FIG. 1. EOB/NR unfaithfulness, Eq. 14, for all the nonspinning configurations of Table $\mathrm{V}$. The outlier behavior of the 0002 configuration is due to large inaccuracies in the postmerger region of the NR waveform, see Fig. 2

is defined as $\left\langle h_{1}, h_{2}\right\rangle \equiv 4 \Re \int_{f_{\min }^{\mathrm{NR}}(M)}^{\infty} \tilde{h}_{1}(f) \tilde{h}_{2}^{*}(f) / S_{n}(f) d f$, where $\tilde{h}(f)$ denotes the Fourier transform of $h(t), S_{n}(f)$ is the zero-detuned, high-power noise spectral density of advanced LIGO [56] and $f_{\min }^{\mathrm{NR}}(M)=\hat{f}_{\min }^{\mathrm{NR}} / M$ is the starting frequency of the NR waveform (after the junk radiation initial transient). Both EOB and NR waveforms are tapered in the time-domain so as to reduce high-frequency oscillations in the corresponding Fourier transforms. Figure 1 shows how $\bar{F}$, computed for $10 M_{\odot} \leq M \leq 200 M_{\odot}$, always remains well below the reference level of $1 \%$, that translates into a negligible loss of events. For all waveforms, we find that $10^{-4} \lesssim \bar{F} \lesssim 10^{-3}$, except for a 32 orbit equal-mass simulation SXS:BBH:0002. The reason for this is that the NR merger and ringdown is rather inaccurate. This can be seen by comparing to $\bar{F}$ for the other two, equal-mass, 28-orbit long, datasets which saturate at the $0.1 \%$ level. The $\bar{F}$ plot itself is not very informative for understanding what is going on. Instead, we find a usual time-domain analysis, comparing the EOB and NR amplitude and frequencies, more illustrative, as shown in Fig. 2. In this figure, as well as below, $\Psi_{22}$ indicates the Regge-Wheeler-Zerilli normalized waveform [57, defined as $\Psi_{22} \equiv \mathcal{R} h_{22} / \sqrt{24}$ where $h_{22}$ is the $\ell=m=2$ strain waveform in $h_{+}-\mathrm{i} h_{\times}=$ $\frac{1}{\mathcal{R}} \sum_{\ell m} h_{\ell m-2} Y_{\ell m}(\theta, \phi)$. The waves are aligned following our usual alignment procedure (described in details in Sec. VA of Ref. [58]) on the following, early inspiral, frequency intervals: $\left[\omega_{L}, \omega_{R}\right]=[0.0248,0.0305]$ for SXS:BBH:0002 and $\left[\omega_{L}, \omega_{R}\right]=[0.0256,0.0317]$ for SXS:BBH:0180. The plot is done versus $u / M$, denoting the NR retarded time at future null infinity. The numerical problems in postmerger phase of SXS:BBH:0002 are strikingly evident in this plot. Still, the EOB/NR agreement is excellent up to the NR merger point, which
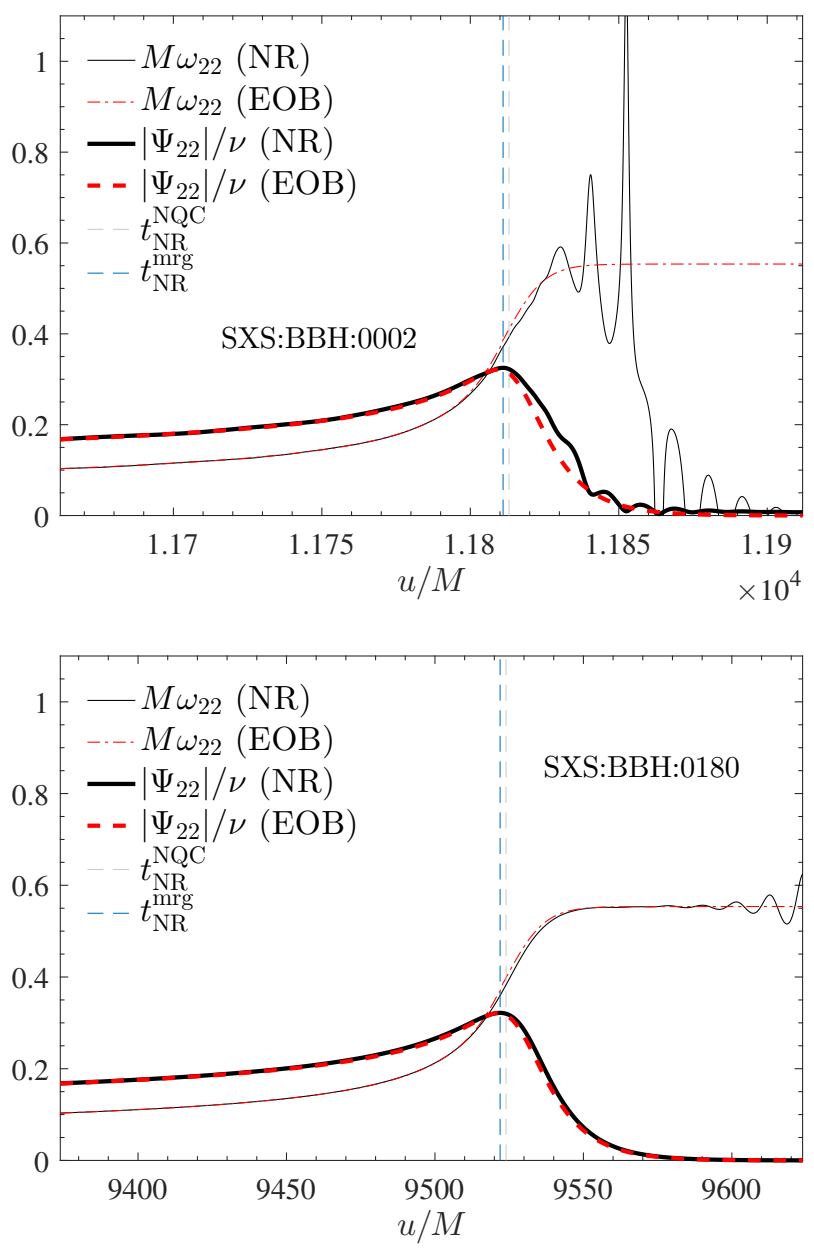

FIG. 2. Equal-mass case: EOB/NR comparisons of amplitudes and frequencies for two equal-mass datasets: SXS:BBH:0002 (32 orbits) and SXS:BBH:180 (28 orbits). The numerical problem after the merger are evident in SXS:BBH:0002. Even if SXS:BBH:0180 was not used in calibration the model in Paper I, it shows excellent consistency with the EOB waveform. The $\mathrm{NR}$ merger time $t_{\mathrm{mrg}}^{\mathrm{NR}}$ as well as the time location of the NQC-extraction point $t_{\mathrm{NQC}}^{\mathrm{NR}} / M \equiv\left(t_{\mathrm{mrg}}^{\mathrm{NR}}+2\right) / M$ are explicitly marked on the plot.

is explicitly indicated on the figure ${ }^{3}$. As mentioned in Paper I, this remains remarkable because the value of $a_{5}^{c}$ was determined exploiting only a 14-orbit-long waveform produced in one of the early SXS runs 33, which is not part of the SXS catalogue but was used in the calibration of previous EOB models [24, 59. All considered, Fig. 1 illustrates that our nonspinning EOB model is rather robust, simple and efficient (thanks to the global fits mentioned above, there is no need of iterating to generate waveforms for production purposes) and the EOB/NR values of $\bar{F}$ are consistent with the expected numerical uncertainty once expressed in terms of this quantity [40].

\footnotetext{
3 The NR point used for NQC determination, at $t_{\mathrm{NQC}}^{\mathrm{NR}}=t_{\mathrm{mrg}}^{\mathrm{NR}}+2$, is also explicitly marked for convenience.
} 


\section{The spinning sector}

Let us now proceed to the spinning sector, that was informed, in Paper I, using a sample of 30 spin-aligned NR simulations. These spinning configurations are listed, for convenience, in Table VI. Starting from this data, Paper I determined the following analytical representation for the NNNLO effective spin-orbit parameter $c_{3}$ as function of the individual spins

$$
\begin{aligned}
& c_{3}\left(\tilde{a}_{1}, \tilde{a}_{2}, \nu\right)=p_{0} \frac{1+n_{1}\left(\tilde{a}_{1}+\tilde{a}_{2}\right)+n_{2}\left(\tilde{a}_{1}+\tilde{a}_{2}\right)^{2}}{1+d_{1}\left(\tilde{a}_{1}+\tilde{a}_{2}\right)} \\
& +\left(p_{1} \nu+p_{2} \nu^{2}+p_{2} \nu^{3}\right)\left(\tilde{a}_{1}+\tilde{a}_{2}\right) \sqrt{1-4 \nu} \\
& +p_{4}\left(\tilde{a}_{1}-\tilde{a}_{2}\right) \nu^{2}
\end{aligned}
$$

where $\tilde{a}_{1,2} \equiv X_{1,2} \chi_{1,2}$. Here we have also defined $X_{1}=$ $\frac{1}{2}(1+\sqrt{1-4 \nu})$ and $X_{2}=1-X 1$. The dimensionless spins are denoted by $\chi_{i} \equiv S_{i} / m_{i}^{2}$ with $i=1,2$. The coefficients for $c_{3}$ are given by 4

$$
\begin{aligned}
& p_{0}=+44.822889, \\
& n_{1}=-1.879350, \\
& n_{2}=+0.894242, \\
& d_{1}=-0.797702, \\
& p_{1}=+1222.36, \\
& p_{2}=-12764.4, \\
& p_{3}=+36689.6, \\
& p_{4}=-358.086 .
\end{aligned}
$$

The function 15 is composed of two parts: a part that accounts for equal-mass, spin aligned binaries, given by a rational function of only $\hat{a}_{0} \equiv \tilde{a}_{1}+\tilde{a}_{2}$, and a further two terms that parametrize the deviations away from the equal-mass, equal-spin regime. The procedure to obtain the fitting parameters $\left\{p_{0}, n_{1}, n_{2}, d_{1}, p_{1}, p_{2}, p_{3}, p_{4}\right\}$ is as follows. First, we focused on the equal-mass, equal-spin case and for each corresponding dataset of Table VI $c_{3}$ wass determined by requiring that the phase difference between the EOB and NR waveforms, aligned in the early inspiral, and computed at NR merger, is comparable or smaller than the corresponding phase uncertainty at the NR merger point; interestingly, it was easy to find "good" values of $c_{3}$ that approximately lie on a straight line (except for very large spins), which eventually yielded to the rational function representation in Eq. (15). A similar procedure was then followed for the unequal-mass, unequal-spin regime, similarly finding good values of $c_{3}$ that were then globally fitted so as to obtain the other two terms of Eq. (15). To give a few more explicit details

\footnotetext{
${ }^{4}$ Note that in Ref. [17] there was a typo in the definition of $c_{3}$ as well as in the value of $p_{0}$, that should read 44.822889 instead of 44.786477 .
}

TABLE I. First-guess values of $c_{3}$ compared with the values obtained from the interpolating fit. (15) for the small sample of NR datasets used to inform the model of Paper I away from the equal-mass, equal-spin limit.

\begin{tabular}{lccc}
\hline \hline$\left(q, \chi_{1}, \chi_{2}\right)$ & $\hat{a}_{0}$ & $c_{3}^{\text {first-guess }}$ & $c_{3}^{\text {fit }}$ \\
\hline \hline$(1,-0.5,0)$ & -0.2500 & 61.5 & 62.61 \\
$(1.5,-0.5,0)$ & -0.300 & 62 & 61.74 \\
$(3,-0.5,0)$ & -0.375 & 63 & 63.69 \\
$(3,-0.5,-0.5)$ & -0.500 & 68 & 66.89 \\
$(5,-0.5,0)$ & -0.4167 & 62 & 62.02 \\
$(8,-0.5,0)$ & -0.4444 & 57 & 57.22 \\
$(1,+0.5,0)$ & +0.2500 & 26.5 & 27.22 \\
$(1.5,0.5,0)$ & +0.3000 & 26 & 28.17 \\
$(2,+0.6,0)$ & +0.4000 & 26.5 & 24.54 \\
$(3,+0.5,0)$ & +0.3750 & 26 & 26.38 \\
$(3,+0.5,+0.5)$ & +0.5000 & 24 & 23.59 \\
$(5,+0.5,0)$ & +0.4167 & 28 & 28.17 \\
$(8,+0.5,0)$ & +0.4444 & 33 & 33.06 \\
\hline \hline
\end{tabular}

about this procedure, Table 1 lists our preferred, "firstguess", values of $c_{3}$ for the 13 asymmetric datasets used as well as the final values provided by the interpolating fit (15). We want to stress that obtaining the first-guess values is very easy due to the controllable physical effect of $c_{3}$, that enters as a parameter in the (resummed) gyro-gravitomagnetic functions of Ref. [51, see in particular Eqs. (41)-(42), (45) and (52). Tuning this parameter essentially corresponds to making the spin-orbit interaction stronger or weaker. In practical terms, this amounts to having a larger or smaller number of GW cycles between the inspiral and merger. Once the NR and EOB waveforms are aligned in the early inspiral, it is easy then to find "good" values of $c_{3}$ simply by hand. This is how the values in the third columns of Table I were actually obtained. In addition to this, the model has to be completed with NQC corrections and an expression of the NQC parameters over the full parameter space is required. Whilst obtaining a direct fit of $\left(a_{i}, b_{i}\right)$, as in the nonspinning case above, would ideally be the best solution (because once the model is NQC-informed by a restricted sample of NR data sets there is no need to iterate outside this calibration domain), in practice it proved difficult to find simple and easy-to-fit behavior, notably due to the large magnitude of the NQC amplitude parameters for equal-mass, large spin binaries, $\chi_{1}=\chi_{2} \gtrsim 0.9$. The reason behind this is that the correction that has to be applied to the EOB waveforms might be rather larger towards merger for these configurations. Since the value of the radial momentum is small, one needs the NQC parameters to increase (they can be up to 4 or 5 times larger than the corresponding values for milder, or antialigned, spins) in order to be able to correct the waveform. As pointed out in Ref. [23], an improved resum- 

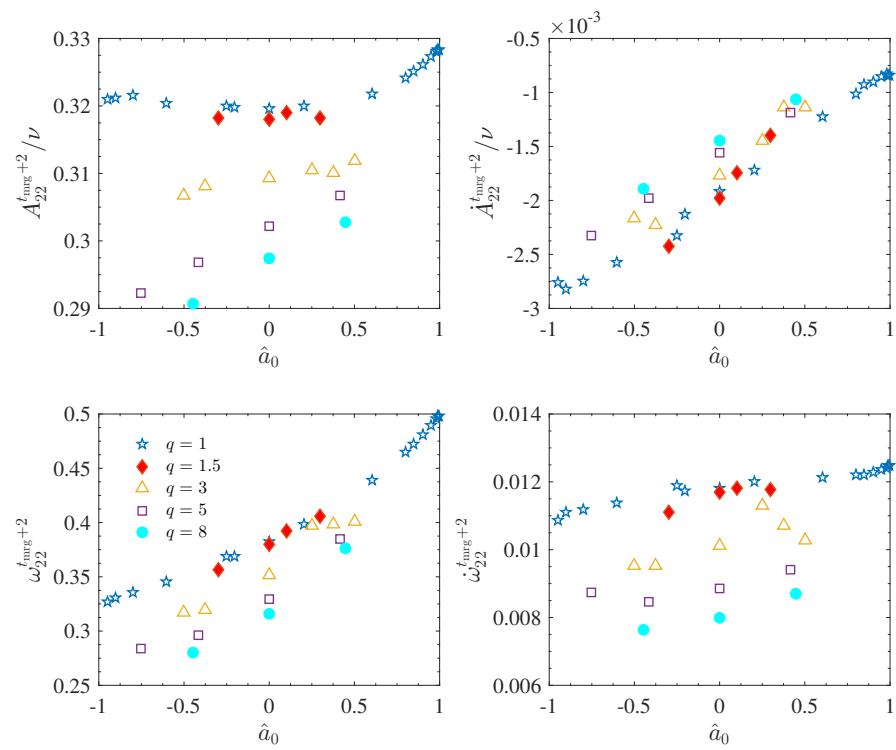

FIG. 3. Dependence of the NR NQC-extraction point on $\hat{a}_{0} \equiv X_{1} \chi_{1}+X_{2} \chi_{2}$. Left panels: amplitude (top) and frequency (bottom) at $t_{\mathrm{NR}}^{\mathrm{NQC}} / M=\left(t_{\mathrm{mrg}}^{\mathrm{NR}}+2\right) / M$. Right panels: corresponding time derivatives at the same time. The four points with $q=1.5$ behave inconsistently with respect to the $q=1$ and $q=3$ data, and are thus discarded to compute the global interpolating fits, see Eq. A2 and Tables II III in Appendix A.

mation of the factorized waveform amplitude can reduce the need of large NQC corrections and, eventually, help in obtaining simpler but accurate global fits of the NQC parameter. Since we postpone this study to future work, we have to find an alternative method to interpolate the NQC parameters outside the domain of calibration. We do so by looking for a global representation of the NR NQC-extraction point. Figure 3 illustrates the behavior of the NR quantities $\left(A_{22}, A_{22}, \omega_{22}, \dot{\omega}_{22}\right)_{t_{\mathrm{NQ}}^{\mathrm{NR}}}^{\mathrm{NR}}$ at the NQC-extraction time, $t_{\mathrm{NQC}}^{\mathrm{NR}} / M=\left(t_{\mathrm{mrg}}^{\mathrm{NR}}+2\right) / M$, where $A_{22} \equiv\left|\Psi_{22}\right| / \nu$ and $\omega_{22} \equiv M \dot{\phi}_{22}$, with $\phi_{22}$ the GW phase and the overdot indicating time derivatives. The figure includes data taken from all configurations used in $\mathrm{Pa}-$ per I plus those extracted from dataset SXS:BBH:0208, with $(5,-0.90,0)$. Inspecting the figure, one immediately notices that the $q=1.5$ points behave somehow differently from the others. This is rather evident in the behavior of $\dot{A}_{22}$ as well as $\omega_{22}$, that are crossing the other $q=1$ and $q=3$ points instead of following the same trend and being in the middle. To avoid inclusion of possible systematics, we then drop all $q=1.5$ datasets. Then we proceed by doing a two-step fit: (i) first, for each value of $\nu$ we fit the points versus $\hat{a}_{0}$ (with polynomials or rational functions, see below) then (ii) we fit the coefficients of this linearly versus $\nu$. In practice, since we have many points for $q=1$, we use two separate fits: (i) a special fit, very accurate, obtained for $q=1$ only, that employs fourth-order polynomials in $\hat{a}_{0}$ for $\left(A_{22}, \dot{A}_{22}, \omega_{22}, \dot{\omega}_{22}\right)$; (ii) a second fit that takes into account all data, but that uses a two-parameters rational function in $\hat{a}_{0}$ for $\omega_{22}$ and quadratic functions in $\hat{a}_{0}$ for the other quantities. More quantitative details about these fits are given in Appendix A. Using this "interpolated" representation of the NR NQC functioning point, $\left(A_{22}, \dot{A}_{22}, \omega_{22}, \dot{\omega}_{22}\right)^{\text {fit }}$, together with the functions for $c_{3}$ and $a_{5}^{c}$ discussed above, we are now in the position of checking the faithfulness of the model all over the spin-aligned configurations available in the SXS catalog. We note that these are actually a few less than those used in Ref. [20, since specific dataset produced for that study are not public yet. We generate EOB waveforms and we compute $\bar{F}$. The EOB waveforms are typically very long, since the relative initial separation is $r_{0} / M=24$ in terms of the EOB radial coordinates. Then, they are cut at some geometric frequency corresponding to a time on the NR time-axis of a few hundreds $M$ 's after the junk radiation. Both NR and EOB waveforms are then tapered before taking the Fourier transform. The total mass of the binary is varied between $10 M_{\odot} \leq M \leq 200 M_{\odot}$; results are shown in Fig. 4. As in the nonspinning case discussed above, the horizontal, dotted, blue line marks the $3 \%$ limit, while the horizontal, black, dotted line marks the $1 \%$ limit. The figure illustrates that: (i) despite relying on the fact that $\left(a_{6}^{c}(\nu), c_{3}\right)$ as well as the NQC and ringdown parameters were informed using the rather small sample of $39 \mathrm{NR}$ waveforms (and notably only the 13 asymmetric NR datasets of Table I, the unfaithfulness is always below $3 \%$ over the whole NR catalog of publicly available 149 spin-aligned waveforms; (ii) more precisely, $\max (\bar{F})$ turns out to be always below $1 \%$ (and when it is so the corresponding line is depicted grey in Fig. (4) except for two dataset that just hit this level $(2,+0.85,+0.85)$ (with $\max (\bar{F})=1.04 \%)$ and $(3,+0.73,-0.85)$ (with $\max \bar{F}=1.05 \%)$, and another two that are larger $(1,+0.90,0)($ with $\max (\bar{F})=1.39 \%)$ and more importantly $(1,+0.90,+0.50)$, that reaches $\max (\bar{F})=2.49 \%$. None of these datasets was available at the time of Paper I; (iii) the results of Paper I, and in particular Fig. 9 there, are essentially confirmed, though just slightly worsened because of the use of interpolating fits for both the NQC point and the postmergerringdown part; (iii) the line depicted in colors in the figure are datasets that were found "problematic" (i.e. with $\max (\bar{F})>3 \%$ ) for SEOBNRv2, as illustrated in Fig. 2 of Ref. 20, but they are below or comparable to the $1 \%$ level here. It is worth commenting in particular the case $(1,-0.90,+0.90)$ : since the spins are equal and antialigned and $q=1$, one can easily see inspecting analytically the EOB dynamics that the spinorbit interaction gets essentially canceled and it is only the effect of the spin-spin interaction (that is subdominant) that cumulates with to the orbital dynamics. Since we see (check Table $\mathrm{V}$ ) that there is a good match between EOB/NR for $(1,0,0)$ one a priori expects that $(1,-0.90,+0.90)$ will work similarly well. Not surprisingly, then, this is what we find in Fig. 4 with $\bar{F}$ of 


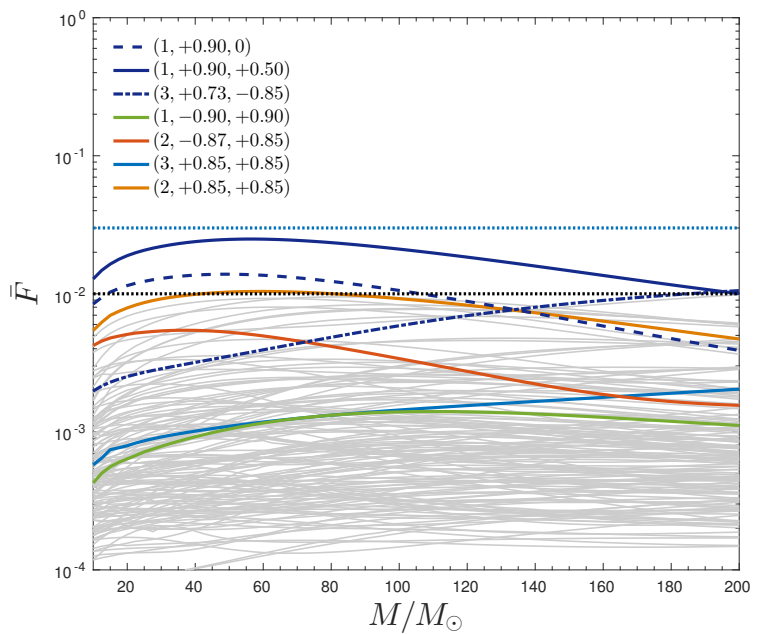

FIG. 4. EOB/NR unfaithfulness computed all over the spinaligned SXS NR catalog. The dark-blue curves are those with $1 \% \lesssim \max (\bar{F})<3 \%$. The grey ones correspond to $\max (\bar{F})<1 \%$. The colored curves are those configurations where $\max (\bar{F})$ computed between the corresponding NR data and the SEOBNRv2 EOB model is larger than $3 \%$ as found in Ref. 20]. Remarkably, except the case $(2,+0.85,+0.85)$ these configurations are all well below the $1 \%$ level.
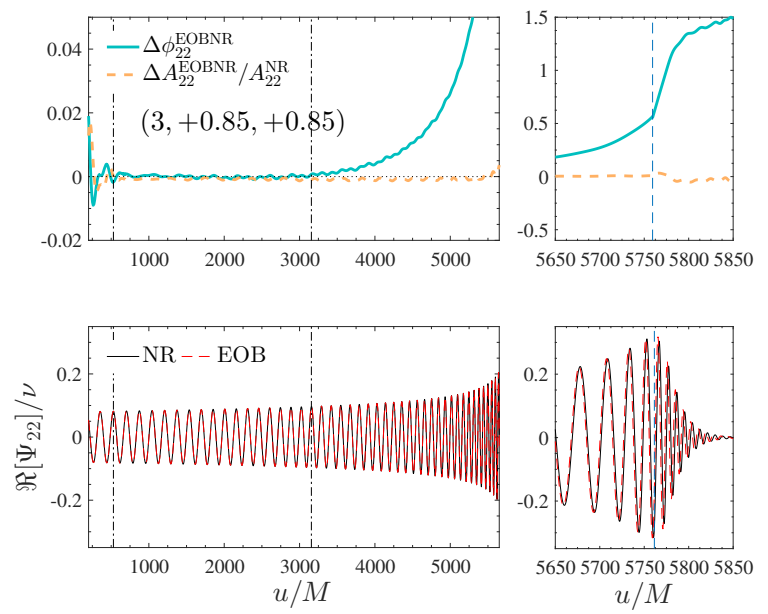

FIG. 5 .

Time-domain phasing comparison for $(3,+0.85,+0.85)$, dataset SXS:BBH:0293. This corresponds to the blue curve in Fig. 4. This phasing result is comparable to the one obtained using SEOBNRv4 (see Fig. 4 of Ref. 20]) although no new calibration of the model of $\mathrm{Pa}-$ per I was needed here.

the order of $0.1 \%$ over the full mass range. This looks rather different from the SEOBNRv2 case, where $\bar{F}$ gets above the $3 \%$ limit, see Fig. 2 of Ref. 20. Before discussing the "outliers" with $1 \% \lesssim \max (\bar{F})<3 \%$, we produce a time-domain phasing comparison, in Fig. 5 , between the EOB and NR waveforms for $(3,+0.85,+0.85)$, dataset SXS:BBH:0293. This plot is the analogous of the time-domain check of SEOBNRv4 model, see Fig. 4 of Ref. [20], although no additional calibration of the model of Paper I was needed to obtain such result. The top panels of the figure show the fractional amplitude difference (orange) and the phase difference (blue), obtained by aligning in time and phase the two waveforms with our standard technique that relies on minimizing the phase difference between the frequencies corresponding to the time-interval marked by the two vertical lines. We note two things: (i) from Table $[\mathrm{IX}$ one sees that the nominal NR uncertainty at merger for this dataset is $\delta \phi_{\mathrm{mrg}}^{\mathrm{NR}}=1.15 \mathrm{rad}$, so the $\mathrm{EOB} / \mathrm{NR}$ phase difference at merger $\Delta \phi_{\mathrm{mrg}}^{\mathrm{EOBNR}}=0.56 \mathrm{rad}$ is fully compatible with this conservative numerical uncertainty; (ii) the derivative of the phase difference in the top-right panel of the figure is discontinuous at merger, where the EOB inspiral-plungemerger description is matched to the phenomenological description of the postmerger-ringdown of Ref. 22. We recall that this phenomenological ringdown representation is built interpolating among the same sample of NR data (plus the case $(5,-0.9,0)$ ), mostly around the equalmass, equal-spin configurations, used in Paper I. In this case, the inaccuracy visible in the time-domain analysis is practically irrelevant for current unfaithfulnesses standard (i.e. $\max (\bar{F})<1 \%$ ), but the same effect can be more important in other configurations, eventually yielding larger values of $\bar{F}$, as we shall see below.

Let us now produce a similar analysis for the four outliers, with $1 \% \lesssim \max (\bar{F})<3 \%$ in Fig. 4 , so to physically understand what is (slightly) inaccurate and eventually improve it. The time-domain waveforms comparisons are shown in Fig. 6. As above, the vertical lines mark the alignment region. One sees that each EOB waveform develops a secular phase difference with respect to the NR one, that develops at latest 2000/3000M before merger. The effect is visually analogous to what appears in Fig. 5 though the main difference is now that the $\mathrm{EOB} / \mathrm{NR}$ phase differences at NR merger, $\Delta \phi_{\mathrm{mrg}}^{\mathrm{EOBNR}} \equiv$ $\phi^{\mathrm{EOB}}-\left.\phi^{\mathrm{NR}}\right|_{t_{\mathrm{mrg}}^{\mathrm{NR}}} \approx(-2.5,-2.2,3.7,-1.4) \mathrm{rad}$, are always larger (except for the the $(3,+0.73,-0.85)$ case) than the numerical nominal NR uncertainties $\delta \phi_{\mathrm{mrg}}^{\mathrm{NR}}=$ $(+1.7,+2.9,+0.3,-0.66)$ This suggests that some strongfield information is (effectively) not incorporated properly in the model. Interestingly, the "worse" result in terms of phasing, for $(2,+0.85,+0.85)$, where the EOB waveform accumulates as much as 3.7 rad of dephasing at merger with respect to the NR one, is actually the one (among these four) with the smallest value of $\max (\bar{F})$, see Fig. 4 . This example illustrates that great care should be put in stating the quality of an analytical waveform model using only $\bar{F}$ as a diagnostics. In fact, although $\bar{F}$ is a useful and essential observable in addressing how good a waveform model is for data-analysis purposes, it might hide important information about the actual compatibility of an analytical (and usually approximate) model versus a NR simulation that is supposed to represent the "exact" result (modulo numerical uncertainties). In other words, if it is true that having a small $\bar{F}(\mathrm{e} . \mathrm{g} \lesssim 1 \%)$ is a necessary criterion to define whether a waveform model is viable for data-analysis purposes (in 

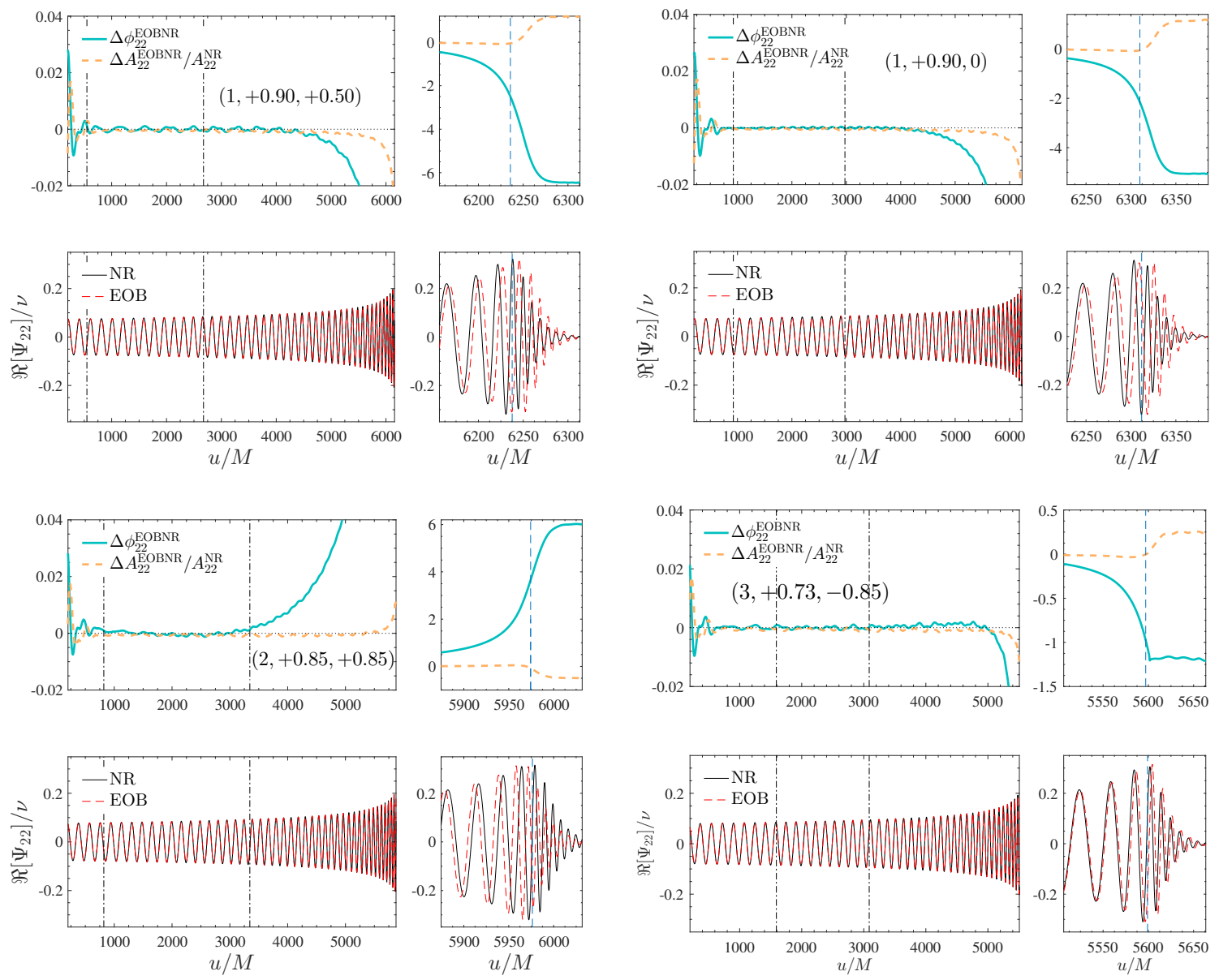

FIG. 6. Time-domain phasing analysis of four outliers (with $1 \% \lesssim \max (\bar{F})<3 \%$ ) in Fig. 4 The waveform are aligned using standard procedure in the time-window indicated on the plots. The phase difference between EOB and NR waveforms, $\Delta \phi^{\mathrm{EOBNR}}$, at merger is typically larger than the corresponding NR error. This calls for the improved determination of $c_{3}$ discussed in Sec. IV

particular for detection, since $\bar{F}<3 \%$ translates into a loss of detections smaller than $10 \%$ [60]), it may not be a sufficiently stringent one to conclude that the analytical model is a fully reliable representation of the physics encoded into a NR waveform.

The opposite situation (i.e., with the phase difference is smaller/compatible with the NR error) is instead found for $(3,+0.73,-0.85)$ configuration, but in this case $\max (\bar{F})$ is just at the border of the usually accepted $1 \%$ unfaithfulness region (the gorwth of $\bar{F}$ is actually due to inaccuracies in the postmerger-ringdown EOB waveform, as we will discuss below and just marginally from the inspiral-plunge). On the contrary, for the other two datasets, the accumulated phase difference is rather large and lies, again, outside the nominal error bar (which is positive), but now this is also clearly mirrored in the values of $\bar{F}$ above the $1 \%$ threshold. Inspecting the plots, one sees that for all datasets except $(2,+0.85,+0.85)$ the EOB waveform is longer than the NR one, with the effect more pronounced in the $q=1$ cases. Within our EOB framework, we physically interpret this fact, in simple terms, by saying that the spin-orbit part of the EOB
Hamiltonian is too strong, i.e. the repulsive effect of the positively aligned spin is too large. By contrast, for $(2,+0.85,+0.85)$ the spin-orbit coupling is too weak and the system plunges faster than it should according to the NR prediction. A simple, new, determination of $c_{3}$ is what it is needed to reduce the EOB/NR phase differences and eventually obtain smaller phase differences as well as smaller values of $\bar{F}$

\section{IMPROVING THE MODEL WITH MINIMAL AMOUNT OF ADDITIONAL NR INFORMATION}

Let us now investigate how the already good results discussed in the previous paragraph can be improved by including a (minimal) additional amount of NR information to the existing EOBNR model. In practice, we inform a new fit for the $\left(p_{1}^{\prime}, p_{2}^{\prime}, p_{3}^{\prime}, p_{4}^{\prime}\right)$ entering Eq. (15) by using the $q=1$ and $q=2$ outliers mentioned above to determine improved value of $c_{3}$ such to reduce the EOB/NR phase difference up to merger and make it closer to the 


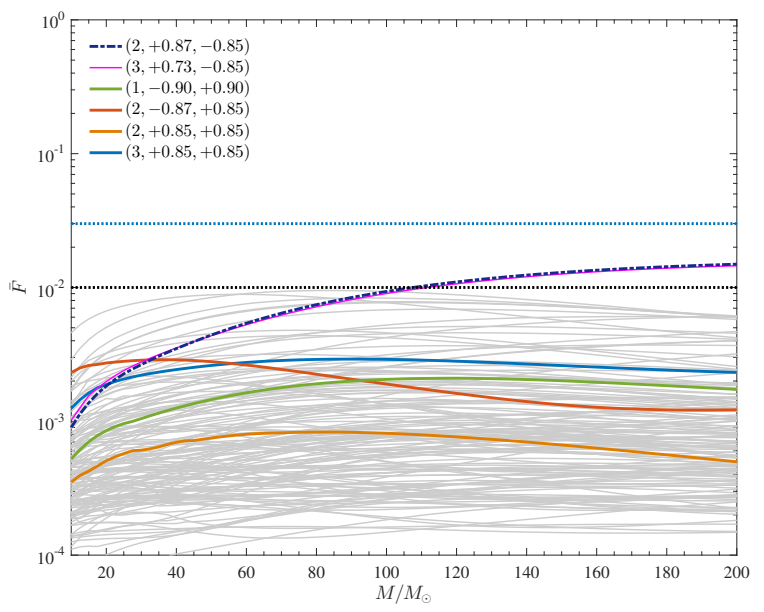

FIG. 7. EOB/NR unfaithfulness obtaned with new determination of of $c_{3}$ yielded by Eqs. (24)- 27). Note the improvement for $(2,-0.87,+0.85)$ (cf. Fig. 4) that is prompted by having determined a better value of $c_{3}$ for $(2,+0.85,+0.85)$. The two outliers (that in Fig. 4 where just below the $1 \%$ level at $\left.M=200 M_{\odot}\right)$ reach now approximately the $1.5 \%$ level because of the relatively poor accuracy of the postmerger interpolating fit.

nominal NR error bar. In addition, we shall also use more NR waveform data to compute an improved interpolating fit of the NR NQC functioning point. By contrast, as mentioned above, here we do not use more NR incorporate more datasets to construct an improved phenomenological description of the postmerger-ringdown waveform (see Ref. [20] in this respect).

\section{A. NR-driven modifications of $c_{3}\left(\nu, \tilde{a}_{1}, \tilde{a}_{2}\right)$}

To determine an improved function for $c_{3}$ we proceed as in Paper I and as briefly reminded above. The EOB and NR waveforms are aligned in the early inspiral and $c_{3}$ is chosen so to reduce the phase difference at merger in a way that $\Delta \phi_{\mathrm{mrg}}^{\mathrm{EOBNR}}$ is compatible (or below) the nominal NR error bar. The fact that, in Fig. 6, the EOB waveform is longer (shorter) than the NR one means that, for these configurations, one has to increase (decrease) the value of the parameter $c_{3}$ with respect to the interpolation provided by Eq. (15) with the coefficients (16)-(23). Following this rationale, one concludes that some new, first-guess, $c_{3}$ values may be $c_{3}=25$ for $(1,+0.90 .0)$ (instead of $c_{3}^{\text {old }}=13.38$ coming from the previous fit), $c_{3}=10.2$ for $(1,+0.90,+0.50)$ (instead of $\left.c_{3}^{\text {old }}=7.97\right)$ and $c_{3}=10.4$ for $(2,+0.85,+0.85)$ (instead of 14.21). We then put these values together with those listed in TableI and a new global fit using the same functional form of Eq. (15) is performed. The equal-mass, equal-spin part of the fit for $c_{3}$ remains untouched, and we only modify the coefficients $\left(p_{1}, p_{2}, p_{3}, p_{4}\right)$, that now read

$$
\begin{aligned}
& p_{1}^{\text {new }}=+978.873 \\
& p_{2}^{\text {new }}=-9456.08 \\
& p_{3}^{\text {new }}=+24304.1 \\
& p_{4}^{\text {new }}=-149.342 .
\end{aligned}
$$

Using such improved analytical representation for $c_{3}^{\text {new }}\left(\nu, \tilde{a}_{1}, \tilde{a}_{2}\right)$ (and without changing anything else with respect to the previous model) we perform a novel computation of $\bar{F}$. The inclusion of the three new "information" points makes $\bar{F}$ always below the $1 \%$ level, though now with the exception of two dataset that reach the $\sim 1.5 \%$ level for $M=200 M_{\odot}$ due to inaccuracies in the modeling of the postmerger phase, as we will illustrate in detail below. Note in addition that for $(1,+0.90,+0.50)$ $\max ((\bar{F}))$ is just below $1 \%(0.89 \%)$ : this was done somehow on purpose to avoid overtuning, by simply requiring that the EOB/NR phase difference at merger, $\Delta \phi_{\mathrm{mrg}}^{\mathrm{EOBNR}}$, is halved with respect to the previous case (see in this respect line 71 and 72 of Table VII . Seen our, very conservative, estimate of the error bar on the NR phasing, we think this is a reasonable choice to improve the $\mathrm{EOB} / \mathrm{NR}$ agreement within the NR phase uncertainty. Let us turn now to discuss in detail the two outliers, $(3,+0.73,-0.85)$ and $(2,+0.87,-0.85)$. The first configuration was already shown to be "quasi-problematic" in Fig. 4 and in the bottom-right panel of Fig. 66. The second one was not labeled as problematic, because $\bar{F}<1 \%$, but in fact in Fig. 4 it is that grey line that closely follows the behavior of the $(3,+0.73,-0.85)$ i.e. it is monotonically growing with $M$ to reach a value just barely smaller than $1 \%$ at $M=200 M_{\odot}$. The inspection of $\bar{F}$ alone seems then to indicate that no improvement is brought here by the new determination of $c_{3}$. This is actually not true and it is hidden by the unfaithfulness plot, that, being given by an integral, hides important local properties of the waveform. To appreciate this, we show the time-domain phasing comparisons in Fig. 8: one sees here that, for $(3,+073,-0.85)$ the previous large EOB/NR phase difference at merger of $\approx-0.66 \mathrm{rad}$ is absorbed by the new value of $c_{3}\left(c_{3}=27.9\right)$ that is smaller than the previous value $\left(c_{3}=22.9\right)$ so to effectively reduce the spin-orbit coupling and make the system plunge faster. Similar consideration apply to the other dataset, where the previous dephasing at merger $\Delta \phi_{\mathrm{mrg}}^{\mathrm{EOBNR}}=-1.8 \mathrm{rad}$ is now reduced to $\approx-0.13 \mathrm{rad}$, wich is compatible with the nominal error bar $\delta \phi_{\mathrm{mrg}}^{\mathrm{NR}} \simeq 0.7 \mathrm{rad}$.

By contrast, in both cases most of the EOB/NR phase difference is accumulated after the merger and mirrors the inaccuracy, in these particular cases, of the interpolating postmerger waveform model that we are using here [29. This is not that surprising, since the model of Ref. 29] was obtained through interpolation of a rather sparse sample of primary ringdown fits that do not include, for example, any $q=2$ waveform data. To prove this is indeed the case, and to remove the inaccuracies coming from the various interpolating fits, we show in 

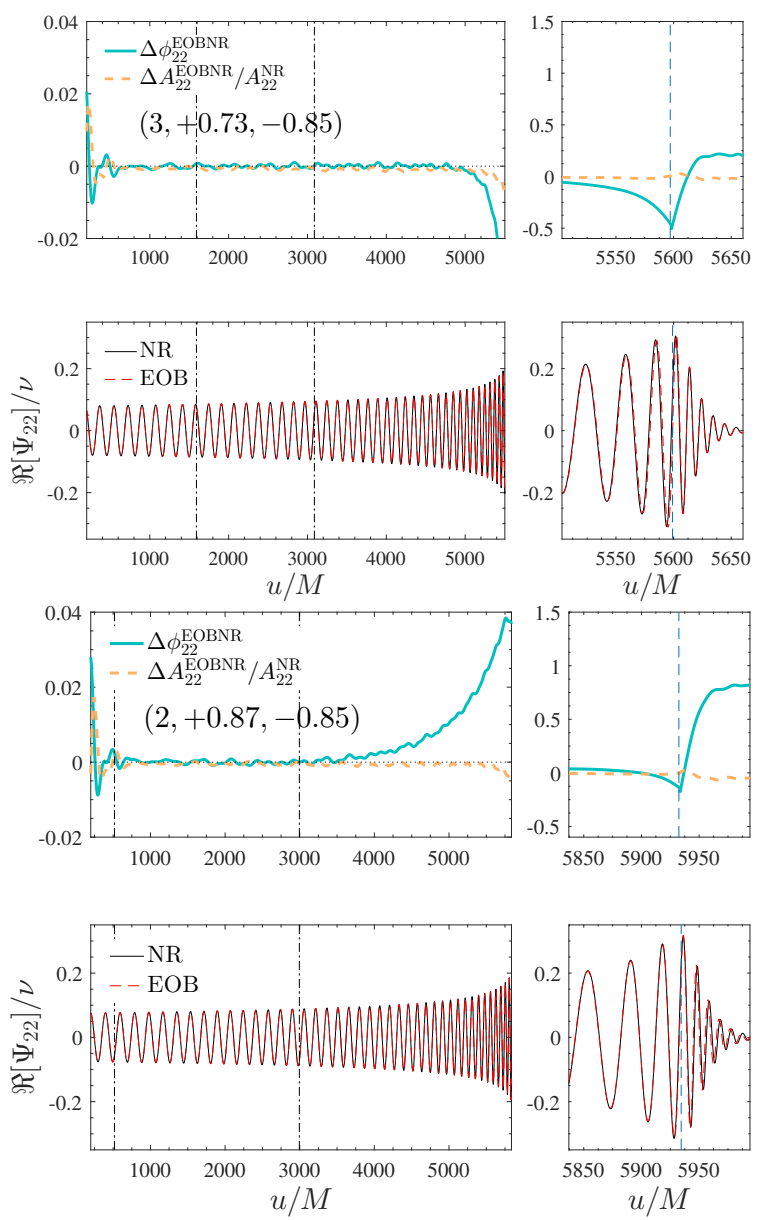

FIG. 8. Effect of the new determination of $c_{3}$ given by Eqs. 24)-27) on $(3,+0.75,-0.85)$ dataset, SXS:BBH:0292, and on (2,+0.87, -0.85), SXS:BBH:0258.

Fig. 9 the level of phasing agreement that can be reached (without changing $c_{3}$ ), for both outliers, using both (i) the exact values for the NR NQC point to determine $\left(a_{i}, b_{i}\right)$ and (ii) the postmerger representation obtained by the primary fit to the NR waveform. The corresponding values of $\bar{F}$ are shown, as solid lines, in Fig. 10 , and remain below $10^{-3}$. This indicates that a fully acceptable model could be obtained by just improving the merger and ringdown description, without changing the value of $c_{3}$. For example, this could be achieved by using more $\mathrm{NR}$ waveform data to produce a more accurate ringdown interpolating fit, as recently done in Ref. 20. Here, we actually try to explore a different route 5 , recalling that the original EOB "recipe" is to construct the ringdown as a superposition of QNMs [2, 6] This basic approach, though in general successful, might be subtle (and in-

${ }^{5}$ As mentioned in Ref. 29], the primary fitting template becomes progressively less accurate when the mass ratio is increased and it should be eventually modified. For this reason, a detailed study is postponed to future work.
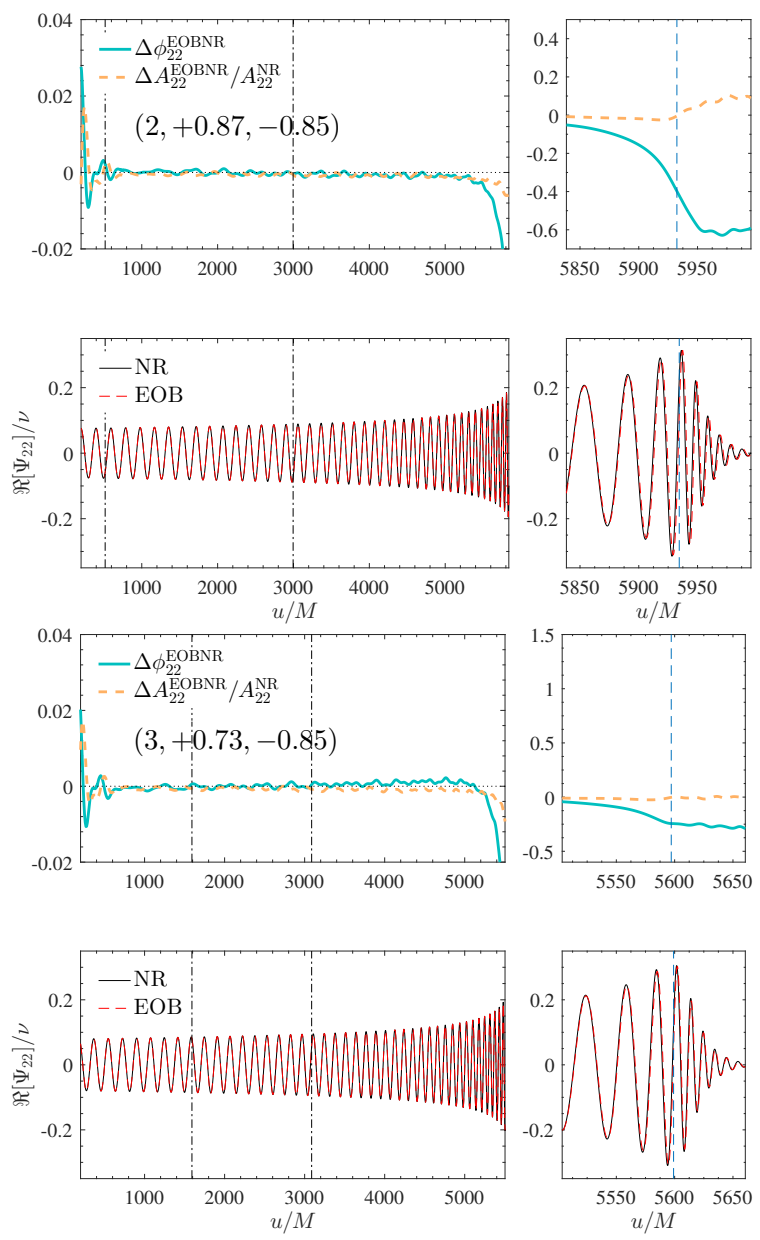

FIG. 9. Same as Fig. 8 but using (i) the "exact", NR values of the NQC functioning point (i.e., not the interpolating fit) and (ii) the description of the ringdown yielded by the primary fit. The value of $c_{3}$ is unchanged with respect to Fig. 8 This result is the best one achievable for this value of $c_{3}$ and yields the solid, thick lines for $\bar{F}$ in Fig. 10.

accurate) for high positive spins, so that pseudo-quasinormal modes frequency were introduced and determined via comparison with NR waveform data [14, 19, 61, 62]. However, in situations where the final black hole spin is not too extreme $\left(a_{f} \lesssim+0.8\right)$, as in this case, the standard QNMs superposition is usually reliable, and offers the possibility of computing accurately the postmerger waveform even without any NR input except the values of the final black hole mass and angular momentum. We then apply the ringdown matching procedure, following Refs. 6, 63, by using $N=8$ QNMs matched to the EOB waveform on a small interval $\Delta t^{\mathrm{QNM}}=0.5 \mathrm{M}$. This approach yields a sufficiently accurate description of the ringdown waveform, as highlighted by the dot-dashed lines in Fig. 10. The figure also illustrates the difference between using the "exact" NR NQC point or the interpolating fit, something that also slightly worsens the global performance. This exercise, done here only on the two outliers, is interesting in that illustrates the power of the 


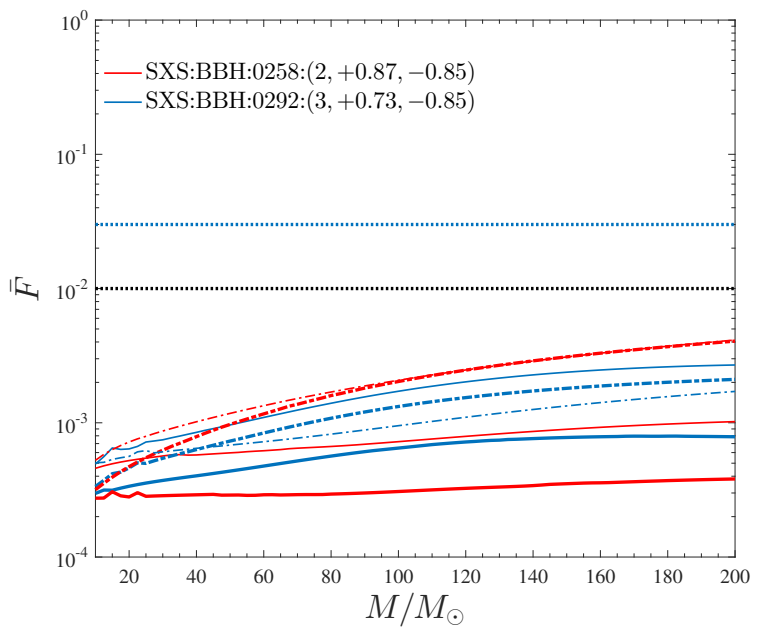

FIG. 10. Improved unfaithfulness for the two outliers of Fig. 7 when the description of the NQC and/or of the postmerger waveform is either improved or changed: (i) thick solid: using the exact NR NQC point and primary NR ringdown fit; (ii) thin, solid: fitted NQC point but primary NR ringdown fit; (iii) thick, dash-dotted: exact NQC point but approximate ringdown description as the superposition of 8 QNMs; (iv) thin, dashed-dotted: fitted NQC point and approximate ringdown description as the superposition of 8 QNMs. This last case can be used as effective replacement of the inaccurate ringdown waveform part highlighted in Figs. 7 and 8 .

simplest approach to ringdown modelization and might eventually turn out useful in regions of the parameter space where the interpolating global fit is clearly inaccurate, provided the final black hole spin is not too high $\left(a_{f} \lesssim+0.8\right)$. The inaccuracy of the phenomenological ringdown description can be easily spotted by inspecting the GW frequency, that develops a large discontinuity at the matching point. In our specific case, such a step in the frequency is behind the cusp appearing in the phase differences Fig. 8. At a practical level, when this inaccuracy is found, the waveform generation algorithm can simply switch from the phenomenological fit to the superposition of QNMs. Although this looks like an easy way out, one has to be aware that a comprehensive estimate of the accuracy of this analytic ringdown representation as function of the binary parameter, and in particular its dependence on the number of QNMs, is currently lacking and will need a dedicated study. In particular, it will be interesting to analyze the performance of the recipe we present here $\left(\Delta t^{\mathrm{QNM}}=0.5 M, N=8\right)$ all over the SXS catalogue.

We finally stress that the results displayed in Figs. 7 and 10 obtained with a new determination of $c_{3}$ with respect of Paper I are certainly not the best possible ones that can be achieved within the current theoretical framework. For pedagogical reasons, here we choose to implement only minimal changes in $c_{3}$ in order to easily accomodate only outliers above $1 \%$ and contemporarily to test the robustness of the model of $\mathrm{Pa}$ per I. We did so by only asking that the phase differ- ence, for those particular datasets, be smaller and/or comparable to the error bar. Actually, other datasets similarly show phase differences at merger larger than $\delta \phi_{\mathrm{mrg}}^{\mathrm{NR}}$, for example $(3,+0.85,+0.85)$, though this is still yielding $\max (\bar{F})<1 \%$. In principle, one could have retuned $c_{3}$ even more with respect to Paper I so to further reduce the $\mathrm{EOB} / \mathrm{NR}$ phase difference (compatibly with the error bar), so to eventually obtain even smaller values of $\bar{F}$. This will be done in a future study that will take advantage of an improved analytical description of the resummed waveform amplitudes [23. The minimal changes we implemented here are mainly aimed at highlighting the simplicity of the tuning procedure and the robustness of the original as well as improved model all over the SXS waveform catalog.

\section{B. Effect of improved fits for the NR NQC-extraction point}

We saw in the previous section that it is possible to improve the faithfulness of the EOB[NR] model of Paper I against the full SXS catalogue by just including three more NR configurations that eventually provide a new fit for $c_{3}$. In addition, the analysis above has also clearly highlighted that, though the phenomenological description of the ringdown of Ref. [29] may become inaccurate already in important regions of the parameter space with $2 \leq q \leq 3$, old ideas and methods for modeling the postmerger-ringdown part prove effective to improve the global accuracy of the model without the actual need of additional NR information. By contrast, the purpose of this Section is to explore how the previous result change by using more NR waveform data to improve one specific part of the EOBNR model, i.e. the determination of the NQC parameters. As an exploratory analysis, we investigate how $\bar{F}$ changes by just using more NR dataset to determine the fit of the NR NQC extraction point. In doing so, both $c_{3}$ and the postmerger description remain unchanged with respect to previous section. We compute here a new fit of $(A, \dot{A}, \omega, \dot{\omega})_{t_{\mathrm{NQC}}^{\mathrm{NR}}}^{\mathrm{NR}}$ using the following data: (i) the same $q=1$ data used before; (ii) all the $q=2$ and $q=3$ equal spin datasets, either aligned or anti-aligned with the angular momentum. We do not consider unequal-spin datasets, except for the ones with $( \pm 0.5,0)$ that were previously included; (iii) we take into account the 4 datasets with $q=5$, with spins $(-0.9,0)$, $(0.0)$ and $( \pm 0.5,0)$; (iv) we drop all $\mathrm{NR}$ waveforms with $q>5$. In particular, we discard all the seven available datapoints with $q=7$, since their behavior versus $\hat{a}_{0}$ appears qualitatively different compared to both $q=5$ and $q=8$ data, and especially inconsistent with them for the longest $(7, \pm 0.4,0)$ waveforms. We will argue in Sec. V below, that it is (partly) related to some (unphysical) drift of the center of mass and, as such, we discard these points to avoid introducing systematics in the fits. This is clearly illustrated in Fig. 11, that displays 

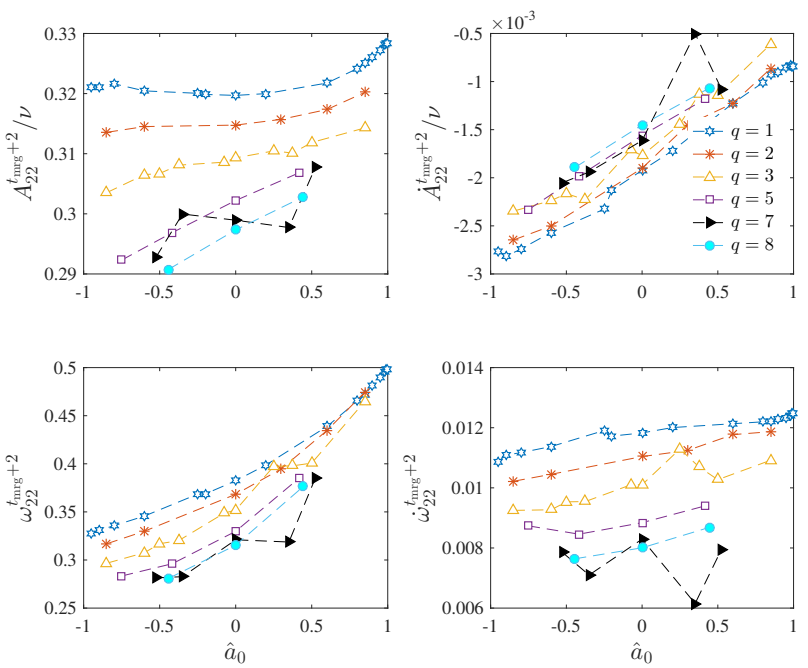

FIG. 11. Behavior of the NR points eventually used to determine improved values of the NQC parameters. These value are taken at $t_{\mathrm{NQC}}^{\mathrm{NR}}=t_{\mathrm{mrg}}^{\mathrm{NR}}+2$ where $t_{\mathrm{mrg}}^{\mathrm{NR}}$ corresponds to the peak of the modulus of the $\ell=m=2$ waveform. Some of the $q=7$ datapoints are clearly inconsistent with the others and are thus not used in the global fits. This weird behavior might be due to some unphysical drift of the center of mass. See Sec. $\mathrm{V}$ for discussion.

$\left(A_{22}, \dot{A}_{22}, \omega_{22}, \dot{\omega}_{22}\right)_{t_{\mathrm{NQC}}^{\mathrm{NR}}}^{\mathrm{NR}}$ versus $\hat{a}_{0}$ for all the configurations we take into account. Interestingly, the plot also illustrates that our previous ansatz for $A_{22}$ and $\dot{A}_{22}$ to be simple quadratic functions of $\hat{a}_{0}$ for $q>1$ is incorrect, since their actual behavior is qualitatively closer to the $q=1$ case. For $q=5$ we cannot make any statement, but since we now have 4 points for this $q$, we can use a cubicin- $\hat{a}_{0}$ ansatz for the amplitude and its derivative instead of the incorrect quadratic one we were obliged to choose before because of lack of datapoints. The explicit values of the fit coefficient (that are used for $q>1$ only, but are determined also including the $q=1$ data) are given in Table IV. Evidently, in order not to lose accuracy, for $q=1$ we still use the previusly determined best fit obtained with 4-th-order-in- $\hat{a}_{0}$ polynomials as given explicitly in Table II. With these improved fits, we regenerate all EOB waveforms for $q>1$ with improved NQC points and recompute the EOB/NR unfaithfulness. The updated result is shown in Fig. 12, where we are also using the QNMs-superposition description for the postmergerringdown for $(2,+0.87,-0.85)$ and $(3,+0.73,-0.85)$. To try to quantify the differences between Figs. 7.10 and 12 , (though one can see that the colored lines have slightly changed), it is convenient to look at histograms of the maximal faithfulness, $\max (F) \equiv \max (\bar{F}))-1$, that we display in Fig. 13. The top panel corresponds to Fig. 4, the medium to Fig. 7 (with the two otuliers, SXS:BBH:0292 and SXS:BBH:025, kept with the inaccurate postmerger-ringdown) and the bottom to Fig. 12 . The values of the medians are respectively, from top to bottom panels, 0.99895, 0.99902 and 0.99908, and are

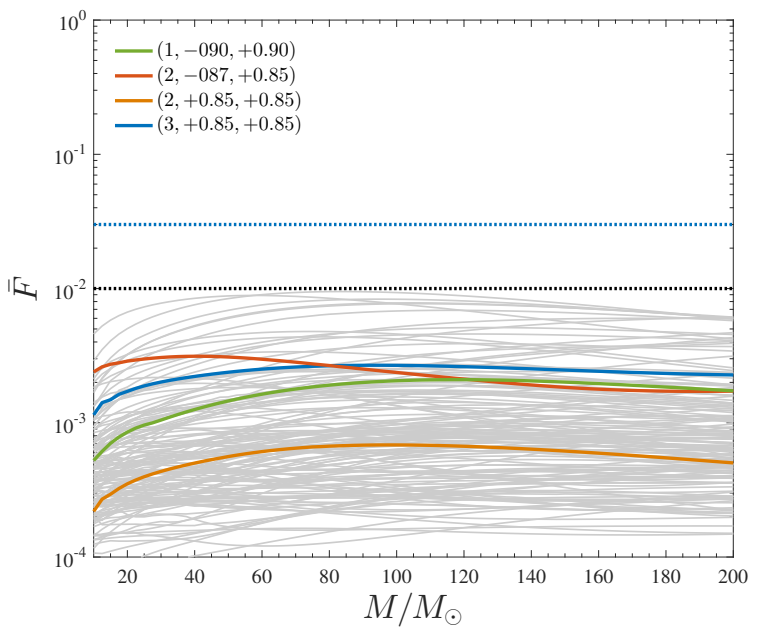

FIG. 12. EOB/NR unfaithfulness with (i) the new fit for $c_{3}$ and (ii) a new interpolating fit for the NR NQC-extraction point that is used for $q>1$ and (iii) the postmmergerringdown description with QNMs for the $(3,+0.73,-0.85)$ and $(2,+0.87,-0.85)$ configurations.

indicated by the vertical dashed lines in the figures. It is interesting to note that the use of a more accurate, and robust, fit of the NQC point that incorporates more NR information, though absolutely needed for theoretical reasons in order to improve the $\mathrm{EOB} / \mathrm{NR}$ consistency at merger, has essentially no impact on this diagnostics.

\section{SXS DATASETS WITH $q=7$}

Let us now come back to the $q=7$ line in Fig. 11 to investigate the probable origin of this behavior. Before doing so, let us however focus on the EOB/NR comparison for $(7,+0.4,0)$, SXS:BBH:0204, whose merger behavior was showing the largest deviation with respect to the global trend of the other datasets. The SXS:BBH:0204 dataset is the longest waveform currently present in the SXS catalog, with approximately 88.4 orbits from the beginning fo the inspiral to merger and ringdown. The standard phasing comparison is illustrated in Fig. 14. The agreement all over is extremely good, with the phase difference barely reaching the 0.1 rad value just a few orbits before merger. However, the top-right panel of Fig. 14 also illustrates rather dramatic phase differences during postmerger-ringdown, with large-amplitude oscillations. The analysis of the waveform amplitude and frequency, top panel of Fig. 15 gives a better understanding of what is going on. First, the modulus looks to have a quite peculiar shape around merger (the peak is wide, with visible oscillations) and also quite relevant oscillations occurr in the frequency during the (expected) ringdown plateau. These oscillations resamble the well-known beating between prograde and retrograde modes, though such an amplitude looks weird here because this type of behavior is typical only when the spins are anti-aligned with 

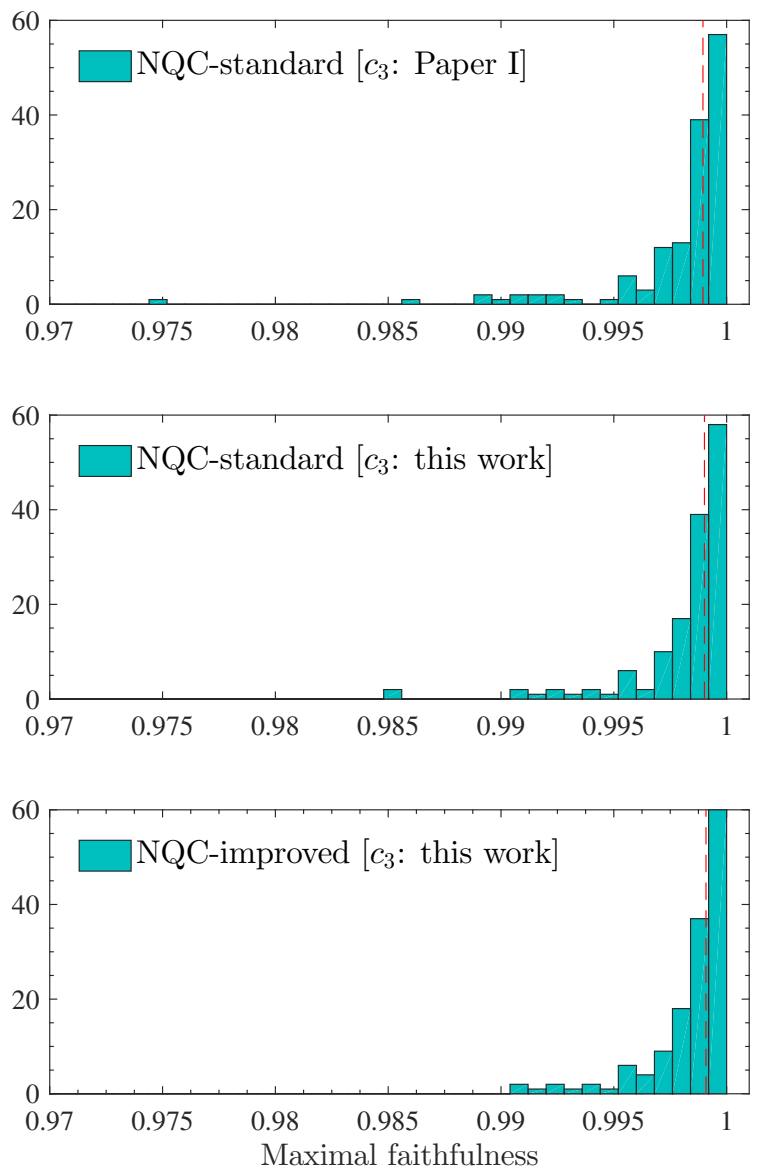

FIG. 13. Improvement of the EOB/NR faithfulness by progressively including more NR information. Top panel: the model of Paper I. Medium panel: effect of the new determination of $c_{3}$. Bottom panel: new determination of $c_{3}$, improved fit for the NQC point and superposition of QNMs to model the ringdown of SXS:BBH:0292 and SXS:BBH:0258.

the angular momentum, and large. So, it seems that there is something unexpected in these waveform, that was also apparent in the NQC plot shown before. These features illustrate clearly that, as with all numerical relativity codes, the use of waveforms requires some care and attention to detail due to the existence and presence of unexpected features. Gravitational-wave data generated from such codes are gauge dependent and may contain artefacts from the numerical schemes used in the construction of the initial data and subsequent evolution of the binary black holes. In particular, the SXS publicly available waveforms are known to contain some features that must be accounted for when comparing to or calibrating semi-analytical waveform models. For example, it was recently highlighted that that SXS waveforms contain unmodeled features arising from the displacement and drift of the center of mass, as seen in Fig. 16 [41] 64.

The presence of such centre-of-mass (CoM) drifts has been associated to the incomplete control of the ADM linear momentum $\mathbf{P}_{\mathrm{ADM}}$ in the construction of initial data 65]. The existence of residual linear momenta leads
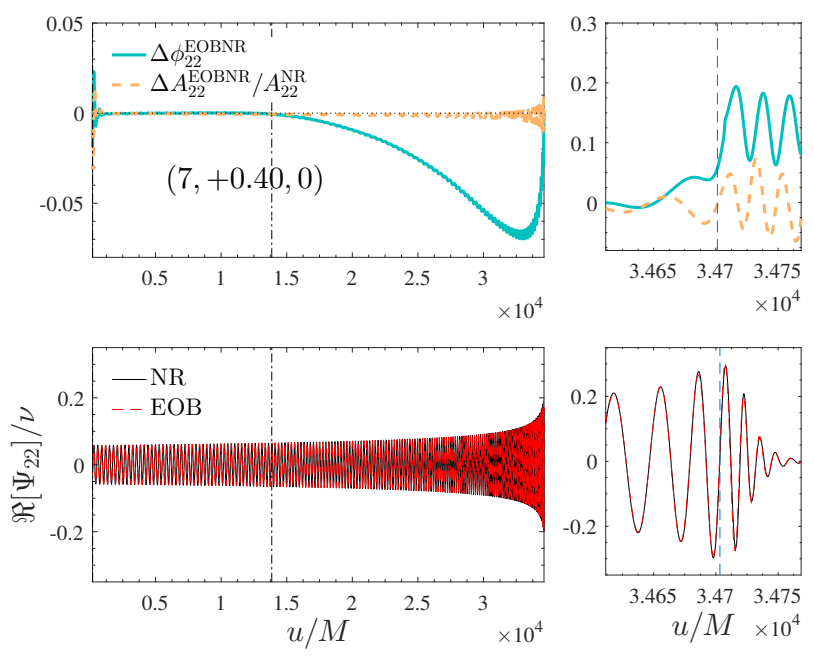

FIG. 14. EOB/NR phasing comparison for the 88.4 orbits long dataset SXS:BBH:0204. It is remarkable how the good phasing agreement is maintained up to merger, notably without using this waveform for calibration.

to an overall drift of the CoM of the binary leading to a number of unmodeled effects. Most notably, as the binary drifts from the origin, the gravitational wave extraction spheres will move off-center with respect to the center-of-mass inducing mode-mixing between the spherical harmonic modes 65. This coupling between the CoM drift and the outer boundary was also identified in the long-duration, nonspinning, $q=7$ SXS simulation [41, 65. SpEC employs constraint preserving outer boundary conditions that are designed to work optimally for lower order spherical harmonics. As the binary drifts or develops non-trivial recoils, the higher order spherical harmonics can become more important and may lead to a runaway acceleration of the CoM [41, 65]. This effect was discussed for the $q=7$, nonspinning, run in which the center-of-mass was observed to increase exponentially with time [4]. The growth rate of the drift was found to depend on the outer boundary radii behaving like $\sigma \propto R^{1.45}$ with timescales on the order of $\sim 2.6 \times 10^{4} M$ for that run. Notably, Ref. [41] pointed out that the com drift eventually yielded effects on the $\ell=m=2$ such to make the merger part rather inaccurate.

A preliminary study of the impact of such drifts on all SXS waveforms was presented in 64. Here it was seen that the $(2,1),(3,3)$ and $(3,1)$ modes exhibited distinct oscillations that were not visible in the other waveform modes. These modes can be seen to have the largest coupling to the dominant $(2,2)$ mode under translations in the $x-y$ plane, suggesting that the unphysical oscillations are a consequence of the mode-coupling induced by the drift of the center-of-mass 64. At merger, it can be seen that power is transferred between modes, leading to corrections above the \%-level to the modeamplitudes. For the SXS:BBH:0004 simulation considered in Ref. 64, the amplitude of the $(2,1)$ and $(3,1)$ 

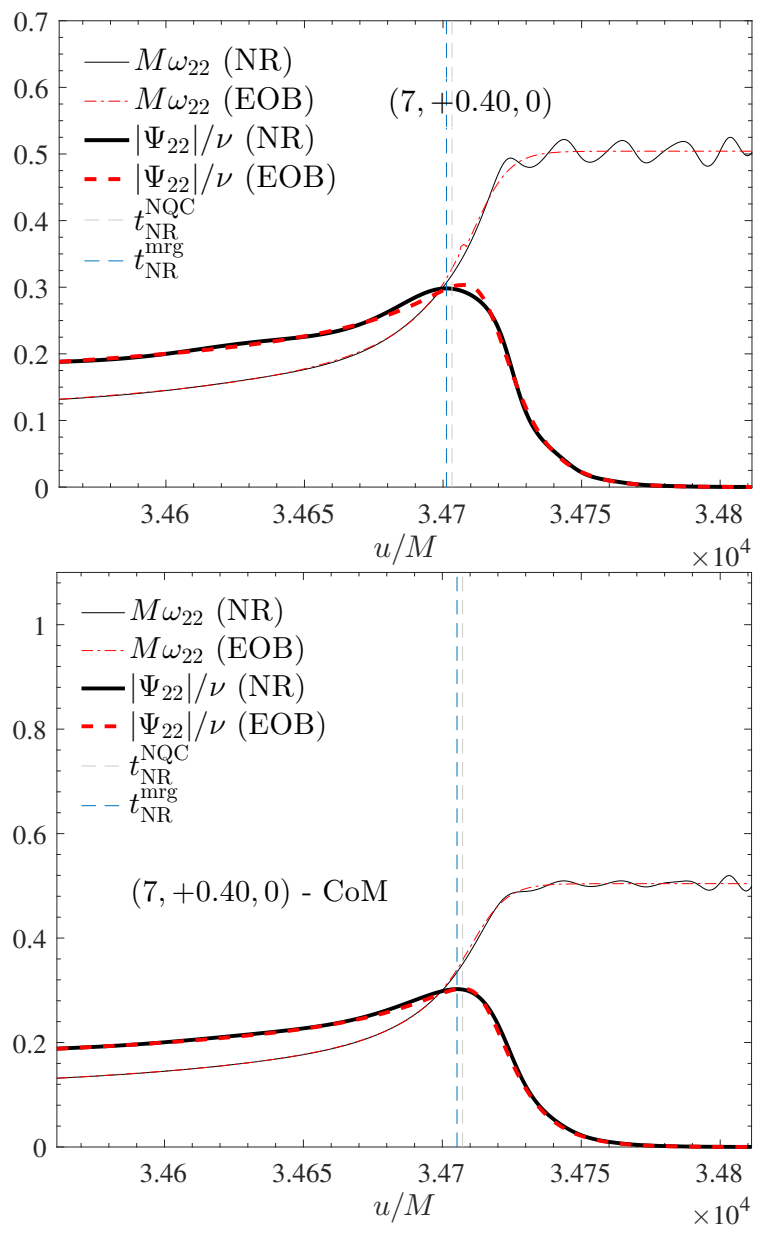

FIG. 15. Effect of the motion of the center of mass on the behavior of the amplitude and frequency of SXS:BBH:0204 of Fig. 14. Top panel: the EOB/NR disagreement between both modulus (around merger) and frequency (oscillations during the ringdown phase) is evident, and mostly due to the unphysical drift of the center of mass of the system. Once this effect is approximately corrected (as illustrated in Fig. 16 below), the NR waveform is visibly more consistent with the EOB one.

modes as a fraction of the $(2,2)$ mode were seen to change by over $1.3 \%$ and the amplitude of $(3,1)$ by $\sim 0.35 \%$. However, Ref. 64] also introduced a framework 6 to account for such drifts by applying a BMS transformation on the Newman-Penrose Weyl scalar $\psi_{4}$ or, alternatively, the transverse-traceless metric perturbation $h$. Schematically, the framework presented in 64 uses the coordinate positions and Christodoulou masses of the black holes from the horizon data in order to minimize the average

${ }^{6}$ An implementation of these BMS transformations can be found in the open-source python code scri: https://github.com/ moble/scri/

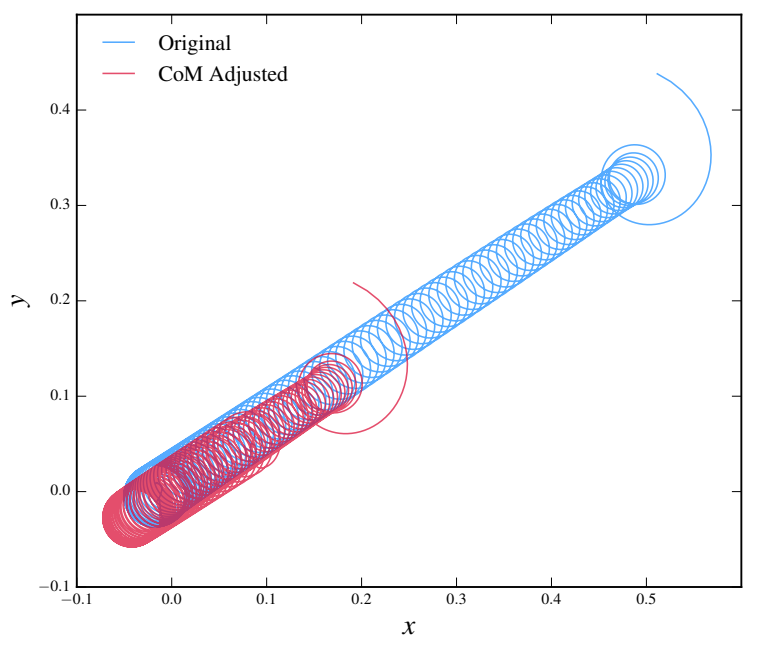

FIG. 16. The impact of the CoM drifts can be mitigated by estimating the optimal boosts and translations from the horizon data, allowing us to correct for such drifts. Here we show the results of the procedure applied to the $(7,+0.4,0)$ SXS:BBH:0204 waveform.

distance between the centre-of-mass and the origin

$$
\Xi(\delta \mathbf{x}, \mathbf{v})=\int_{t_{i}}^{t_{f}}\left|\mathbf{x}_{\mathrm{CoM}}-(\delta \mathbf{x}+\mathbf{v} t)\right|^{2} d t .
$$

This allows one to estimate the optimal translation and boost $(\delta \mathbf{x}, \mathbf{v})$ that can be used to remove drifts from the numerical waveforms. Although the initial offset of the SXS waveforms can be small, the length of the simulations means that even small boosts can grow into translations that dominate over the initial offsets. As noted in [64, the procedure detailed above will be susceptible to a number of gauge effects but does remove or mitigate unphysical effects that are not anticipated based on our understanding of analytical waveforms.

An updated implementation of the quasi-equilibrium conformal thin-sandwhich initial data formalism was introduced in 65] partly with the aim of controlling the spurious residual linear momenta present in the initial data. However, for the current waveform catalog and subsequent simulations that retain significant residual momenta or develop non-trivial recoils, the waveforms can be post-processed to help eliminate or mitigate such initial offsets and overall drifts.

Coming back to the long 88.4 orbits SXS:BBH:0204 waveform, it might be possible that the strange feature in the modulus as well as the oscillations in the frequency during ringdown are related to such unphysical drifts of the CoM. Before entering this discussion let us recall that: (i) there is no mention of the existence of these effects in papers of the SXS collaboration where this waveform dataset was presented for the first time; (ii) a simulation with the same parameters but smaller initial separation, corresponding to 58 orbits up to merger, 


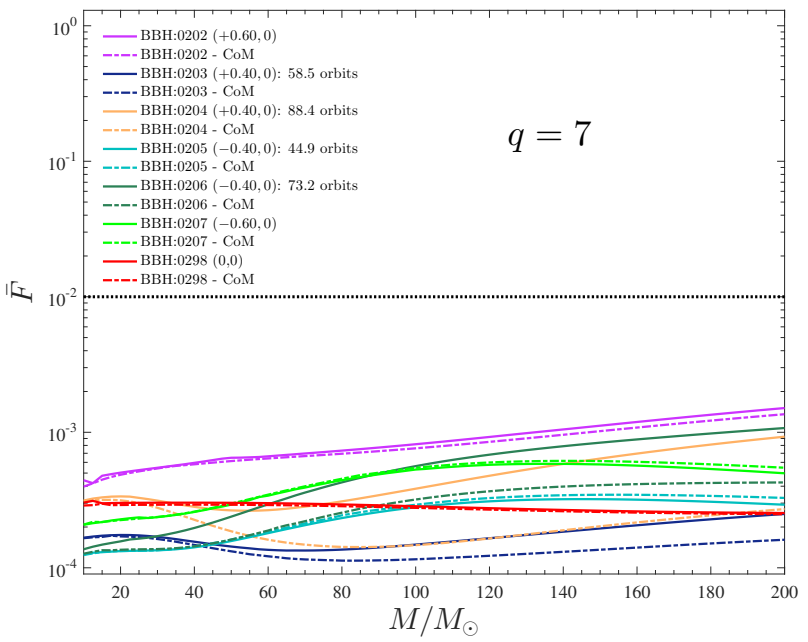

FIG. 17. EOB/NR unfaithfulness for $q=7$ binaries. The removal of the CoM drift using Eq. 28 visibly reduces $\bar{F}$ for $( \pm 0.4,0)$ datasets.

SXS:BBH:0203, yields a waveform where the frequency is perfectly flat (no oscillations) and the modulus has a similarly smoother shaper much closer to the EOB one. The SXS:BBH:204 waveform without the com drift yields the bottom panel of Fig. 15. the modulus is fully consistent, at merger, with the EOB one; the small-amplitude oscillations in the late inspiral part of the modulus have disappeared as well as those in the post-merger part of the frequency.

We have verified that the same picture is true for the other $q=7$ datasets although the importance of the com drift depend on the simulation under consideration. For example, it is visible for the $(7, \pm 0.4,0)$ data, more evidently for SXS:BBH:0204 and SXS:BBH:0206, and less, but still not negligibly, for the shorter SXS:BBH:0203 and SXS:BBH:0205. By contrast, the effect is practically absent for the $(7, \pm 0.6,0)$ datasets, SXS:BBH:0202 and SXS:BBH:0207. This analysis justifies our choice of not using $q=7$ data for the NQC fits. Finally, it is interesting to evaluate the impact of the com drift in the usual EOB/NR unfaithfulness comparison, Fig. 17, where one sees clearly the effect of the removal of the com drift on the longest waveforms. By contrast, as mentioned above, the correction is (essentially) ineffective for the shortest one, except for SXS:BBH:0203, where some effect is seen. The fact that for SXS:BBH:0202 the unfaithfulness $\bar{F}$ is found to grow monothonically is mirroring slight inaccuracies in the postmerger interpolating fit, analogously to what discussed in Fig. 7, as well as the absence of prograde $(m<0)$ modes (that are somehow present for the negative spin datasets, see Ref. 29]) in the modelization of the ringdown. Although the values of $\bar{F}$ in Fig. 17 are well below the standard target of $1 \%$, it is remarkable that they can be improved not by changing the analytical model, but rather correcting systematics in the NR waveforms.

\section{BAM SIMULATION WITH $q=8$}

To anticipate future work, let us finally explore how our EOB model performs in a region of the parameter space marginally outside the one covered by the SXS waveform catalog. To do so, we compare the EOB prediction wth one waveform with physical parameters $(8,+0.85,+0.85)$ that was previously produced using the BAM code 44-46 and employed to calibrate the IMRPhenomD model [13, 48. This waveform is the same mentioned in Ref. [20] (at one resolution with eccentricity of $1.2 \times 10^{-2}$ ) and was checked to be in excellent agreement with an improved one (smaller eccentricity, higher resolution) obtained simulated using the Einstein Toolkit. Following this statement of Ref. 20] we can thus consider this BAM waveform accurate enough for testing the $\mathrm{EOB}[\mathrm{NR}]$ model we present here. To be clear, we deal now with the new determination of $c_{3}$, but we rely on the interpolating fit for the NQC-extraction point of Section IIIC and not the one of Section IV B. We have verified that the benefits of using the improved fits are negligible.

Figure 18 illustrates the EOB/NR unfaithfulness obtained with this model (dashed, dark-grey, line) that is starting above $1 \%$ and eventually crosses the $3 \%$ level. Note also that this waveform is very short, so that the $\bar{F}$ plot largely emphasizes inaccuracies of the late-inspiral, merger and ringdown. The result of Fig. 18 is better understood by comparing the EOB and NR instantaneous GW frequencies, $\omega_{22}$, Fig. 19, after aligning the waveforms on the frequency interval $\left[\omega_{L}, \omega_{R}\right]=[0.3,0.35]$, i.e. during the plunge phase. The interpolating postmerger fit provides a frequency at matching point that is too large with respect to the actual value. This eventually means that the EOB and NR waveforms dephase very fast after the matching point, and explains the growth of $\bar{F}$ when $M$ is increased and the merger part of the waveforms progressively moves towards the most sensitive window of the detector. However, Fig. 19 also illustrates that the issue comes completely from the postmerger-ringdown part, while the behavior of the EOB frequency provided by the combination of $c_{3}$ and of the NQC fit (that, we remind, were determined independently of this dataset) is fully consistent with the corresponding NR one. To highlight this better, in Fig. 19 we show, as a red line, the description of the postmerger waveform obtained using the primary fit instead of the interpolated one. Not surprisingly, in this case $\bar{F}$ also drops down to the $10^{-3}$ level (see Fig. 18). This fact indicates that the most urgent need to improve our model is to build improved interpolating fits of the postmerger waveform, following [29, that crucially incorporate NR waveform data with large values of $q$ and large spins. Finally, it is interesting to explore what can be achieved in this case by using the old-fashioned description of the ringdown as a superposition of QNMs with constant coefficients. As above, we use $N=8$ QNMs and $\Delta t^{\mathrm{QNM}}=0.5$. Figures 1819 show that an improvement with respect to the interpolating fit 


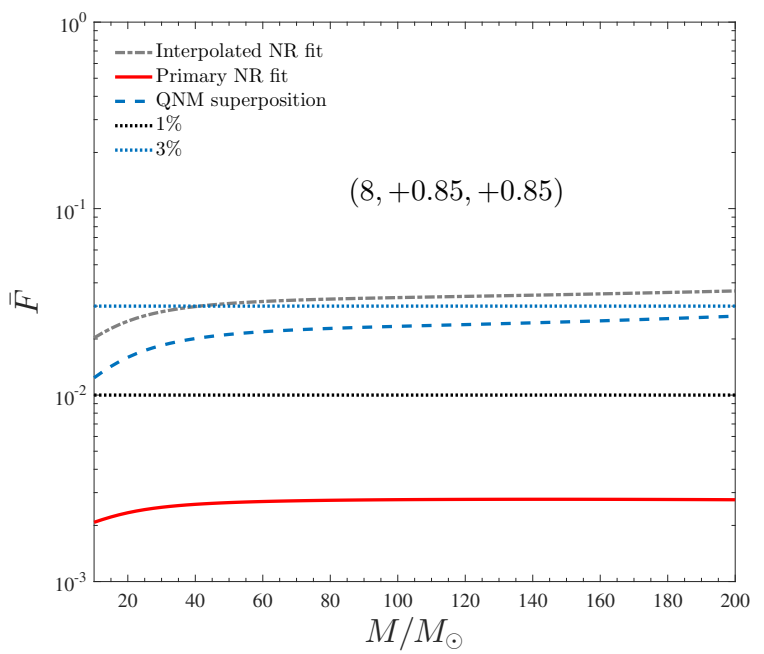

FIG. 18. Unfaithfulness between EOB and the NR dataset $(8,+0.85,+0.85)$ obtained using the BAM code. The growth of $\bar{F}$ is mirroring the inaccurate representation of the ringdown yielded by the interpolating postmerger fits of Ref. [29]. If the primary NR fit is used, the model yields a more than acceptable result.

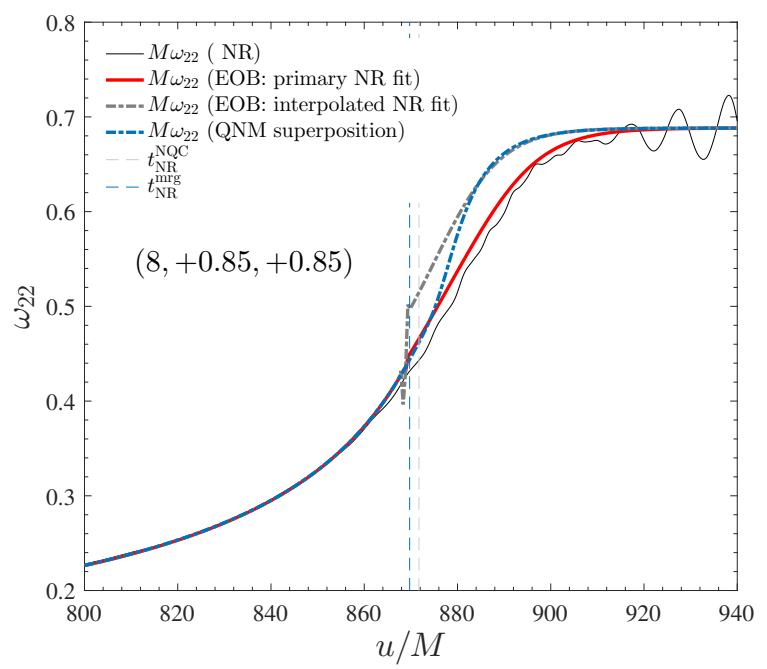

FIG. 19. Understanding the inaccuracy of the EOB waveform highlighted in Fig. 18. EOB/NR comparison between the gravitational wave frequency obtained with different representations of the postmerger phase. If the superposition of $8 \mathrm{QNMs}$ is unable to correctly reproduce the correct frequency behavior, there is no problem of doing it via the primary fit following Refs. 52 .

case, though the frequency disagreement close to the onset of the ringing of the fundamental model is still large enough that the unfaithfulness is between $1 \%$ and $3 \%$. Although this straightforward approach does not yield a fully satisfying solution to the problem, it still represents a reasonable, and no costly, procedure that can be applied when the interpolating fits looks clearly inaccurate. Also, this is the typical situation that could be solved by the implementation of a "pseudo-QNM" frequency in

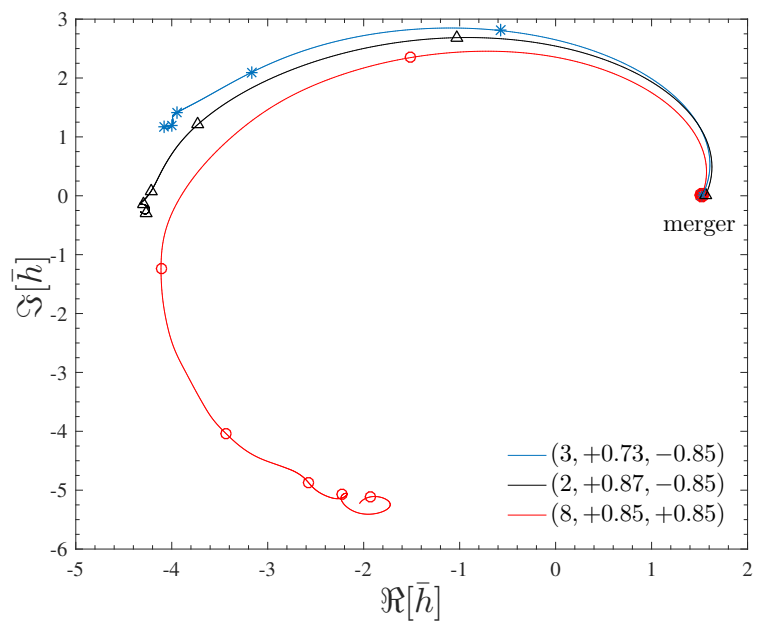

FIG. 20. Behavior of the QNM-rescaled postmerger waveform $\bar{h}$, in the complex plane, for the three datasets considered discussed in the text. Each marker indicates intervals of $10 M_{f}$ after the merger point of the respective waveform. The fact that the $(8,+0.85,+0.85)$ curve rotates by almost $2 \pi$ prevents it from being fully represented by a superposition of QNMs. The nonfactored waveform is dominated by the fundamental QNM only when the markers are approximately superposed. Note in this respect that the BAM waveform is more noisy than the SXS ones.

order to bridge the gap between the merger frequency $M \omega^{\mathrm{mrg}} \approx 0.45$ and $\omega_{8} \simeq 0.6$, that is the frequency of the eight overtone, i.e. the lowest frequency included in the QNMs template.

Let us finally comment on the reason why the ringdown description as a linear combination of QNMs with constant coefficients was rather accurate for $(2,+0.87,-0.87)$ and $(3,+0.73,-0.85)$ while it is not for $(8,+0.85,+0.85)$. This is done, following Ref. [52, notably Sec. III and Fig. 2 there, by inspecting the diagnostics given by the postmerger waveform where the fundamental mode is factored out, $\bar{h} \equiv e^{\sigma_{1} \tau+\mathrm{i} \phi_{22}^{\mathrm{mrg}}}$. Here, $\sigma_{1} \equiv \omega_{1}+\mathrm{i} \alpha_{1}$ is the complex frequency of the fundamental mode, $h \equiv h_{22}$ is the waveform, $\tau \equiv\left(t^{\mathrm{NR}}-t_{\mathrm{mrg}}^{\mathrm{NR}}\right) / M_{f}$ is the dimensionless time parameter that counts time in units of the final $\mathrm{BH}$ mass $M_{f}$, and $\phi_{22}^{\mathrm{mrg}}$ is the value of the phase at merger (so that $\bar{h}(0)$ is the real amplitude of the waveform $h$ at merger. The rescaled waveform $\bar{h}$ is considered as a complex number. It is shown in Fig. 20 for the three NR waveforms considered here. It was noted in Ref. 52] that one can represent the ringdown as a linear superposition of QNMs with constant coefficients only if $\bar{h}$ describes an approximately straight line in the complex plane. This is so because the complex frequency differences $\sigma_{21} \equiv \sigma_{2}-\sigma_{1}, \sigma_{31} \equiv \sigma_{3}-\sigma_{1}$, $\ldots$ are approximately real and positive, especially for high spins. By contrast, when the spin of the final $\mathrm{BH}$ gets high, the early postmerger part $\bar{h}$ is seen to strongly rotate, which means that it cannot be represented as a standard superposition of QNMs. This was discussed at length in Ref. [52, that was focusing in par- 
ticular on the case $(1,+0.97,+0.97)$ as an example of waveform where one cannot use a linear combination of QNMs with constant coefficients starting from the peak of the waveform (see also Ref. 66, 67, for similar conclusions obtained though using different diagnostics.) Inspecting $\bar{h}$ for the three datasets considered here, one finds that $\bar{h}$ is relatively "straight" for $(2,+0.87,-0.87)$ and $(3,+0.73,-0.85)$, that correspond to $a_{f} \approx 0.84$ and $a_{f} \approx 0.836$ respectively, while for $(8,+0.85,+0.85)$, that yield to $a_{f} \approx 0.89$, one has a situation much closer to the $(1,+0.97,+0.97)\left(a_{f} \approx 0.945\right)$ case discussed in Ref. [52]. The precise and quantitative understanding, using $h$, of which region of the parameter space can be easily represented by a superposition of QNMs and when one has to resort to alternative description needs precise investigations. As a rule of thumb, when the final $\mathrm{BH}$ spin is not too extreme, and thus the postmerger-ringdown waveform relatively short, resorting to a superposition of QNMs usually give reliable results. Typically, this is the case when the black holes are nonspinning or the spins are anti-aligned with the orbital angular momentum. One the contrary, when the transition from inspiral to plunge and merger is more adiabatic, e.g., high spins aligned with the orbital angular momentum, the postmerger part brings many cycles and this is usually inaccurately captured as a superposition of QNMs because of the "rotation" of $\bar{h}$ described above. However, there might be many intermediate situations (e.g., unequal masses, one spin up and one spin down) where the straightforward QNMs superposition can be accurate enough for analytical modeling purposes. We plan to analyze these situations quantitatively in future work.

\section{CONCLUSIONS}

We have tested the SEOBNR_ihes EOBNR model introduced in Ref. 17] (Paper I) on the very large sample of $149 \mathrm{NR}$, spin-aligned, datasets publicly available through the SXS catalog [28]. Of these 149 datasets, 96 became available only recently, after October 31st 2016. Our most relevant findings can be summarized as follows:

1. We found that, despite the rater small number (40) of datasets that were used to tune the model of Paper I, without any additional change or tuning the model is found to perform rather well all over the full NR sample of 149 configurations. Looking as usual at the EOB/NR unfaithfulness $\bar{F}$, with total mass $M$ varying between $10 M_{\odot}$ and $200 M_{\odot}$, we find that $\max (\bar{F})<1 \%$ for all datasets except for four outliers that are in the range $1 \% \lesssim \max (\bar{F})<$ $3 \%$. This result by itself probes the robustness of the theoretical construction of Ref. [51] as well as of the NR information procedure of Paper I. In addition, this is also illustrates that only a rather small amount of NR waveform data is effectively necessary to construct a model that would in principle yield an acceptable loss of events $(<10 \%)$ if used for detection purposes.

2. We then proceeded by applying minimal changes to the model in order to improve it even further, aiming at having $\max \bar{F}<1 \%$ all over the SXS catalog. To do so, we added three more simulations to those used to inform the model in Ref. [17, so to provide an improved determination of the next-to-next-to-next-to-leading spin-orbit effective spin-orbit parameter $c_{3}$ that slightly modified the EOBNR spinning waveform of Paper I generated away from the equal-mass, equal-spin regime. In practice, this amounts in changing only four numerical coefficients in the global fit of $c_{3}$ with respect to Paper I, that are listed in Eqs. 24)-(27). The new evaluation of the unfaithfulness is again below $1 \%$ except now for two (more) outliers that reacj the $1.1 \%$ level at $200 M_{\odot}$. We show that this is only due to the somehow inaccurate representation of the postmerger-ringdown waveform by means of the phenomenological fit of Ref. 29] that was extrapolating from a small sample of sparse NR waveforms to the full parameter space. When a more accurate representation of the postmerger phase is used, e.g. using a standard superposition of $N=8$ QNMs, values of $\max (\bar{F})$ lower than $1 \%$ are easily recovered (see Fig. 10).

3. We analyzed in detail the $q=7$ waveform data included in the SXS catalog and concluded that the longest waveforms presents features, around merger, that are due to the spurious motion of the center of mass and, as such, should probably not be used to inform the model. Still, once the unphysical features are (approximately) removed, the consistency of the NR waveform with the EOB prediction is fully recovered, with values of $\bar{F}$ well below the $0.1 \%$ level.

4. We also compared the EOBNR prediction against a BAM waveform with parameters $(8,+0.85,+0.85)$ that was previously used in Ref. [20]. We find, again, that the Achille's heel of the model is represented by the postmerger-ringdown interpolating fit, while the EOB/NR compatibility up to merger is more than satisfactory (see Fig. 19). In this particular case, due to the large value of the spin of the final $\mathrm{BH}$, the standard superposition of QNMs is unable to reliably represent the postmerger phase, though it is able to lower $\bar{F}$ below the $3 \%$ level. Our analysis suggests that the most urgent needs to improve our model is to produce more accurate representations of the postmerger waveform in order to improve the postmerger-ringdown models of Refs. [20, 29], in particular for large mass ratios and large spins. The old-style ringdown representation as a superposition of QNMs might still be effective and useful in some corners of the parameter space, 
but it will not work in more extreme regimes were the NR information is crucial. It would then be useful to have targeted, possibly short and inexpensive, NR simulations with the precise aim of accurately representing the postmerger-ringdown waveform all over the parameter space.

\section{ACKNOWLEDGMENTS}

We are grateful to M. Hannam and S. Husa for lending us their $(8,+0.85,+0.85)$ BAM waveform discussed in Sec. VI.

\section{Appendix A: Fits of the NR points used to determine NQC parameters}

The starting points for the iterative determination of the NQC parameters are obtained as follows. Firstly, the waveform amplitude, frequency and their time derivatives, $\left(A_{22}, \dot{A}_{22}, \omega_{22}, \dot{\omega}_{22}\right)_{t_{\mathrm{NQ}}^{\mathrm{NR}}}^{\mathrm{NR}}$, are extracted from the $\mathrm{NR}$ waveform at the time on the $\mathrm{NR}$ axis $t_{\mathrm{NQC}}^{\mathrm{NR}} / M \equiv$ $\left(t_{\mathrm{mrg}}^{\mathrm{NR}}+2\right) / M$. From these values, one performs fits against the effective spin

$$
\hat{a}_{0}=X_{1} \chi_{1}+X_{2} \chi_{2}
$$

Then, the coefficients of this fit are fitted as linear functions of $\nu$. The dependence on $\hat{a}_{0}$ chosen depends on the quantity as well as on the number of datapoints used. For example, for $q>1$ we fit the points with a rational function of $\hat{a}_{0}$ that yields

$$
\omega_{22}^{\mathrm{NQC}}=\frac{0.45584139 \nu+0.27315247}{1+(0.75276414 \nu-0.40081625) \hat{a}_{0}}
$$

when fitting the points in Fig. 3, or

$$
\omega_{22}^{\mathrm{NQC}^{\prime}}=\frac{0.46908067 \nu+0.27022141}{1+(0.64131115 \nu-0.37878384) \hat{a}_{0}}
$$

for fitting the points in Fig. 11. For $q=1$ we just adopt fourth-order polynomials, and the outcome of the fit is given in Table II. The fits for $q>1$ corresponding to the datapoints used in Fig. 3 are given in Table III the alternative NQC-point fits that use more NR points, as illustrated in Fig. 11 and related text, are given in Table IV.
[1] A. Buonanno and T. Damour, Phys. Rev. D59, 084006 (1999)

[2] A. Buonanno and T. Damour, Phys. Rev. D62, 064015 (2000)

[3] T. Damour, Phys. Rev. D64, 124013 (2001)

[4] A. Buonanno, Y. Chen, and T. Damour, Phys. Rev. D74, 104005 (2006), arXiv:gr-qc/0508067.

[5] T. Damour, E. Gourgoulhon, and P. Grandclement, Phys.Rev. D66, 024007 (2002), arXiv:gr-qc/0204011 [grqc].

[6] T. Damour and A. Nagar, Phys. Rev. D76, 064028 (2007), arXiv:0705.2519 [gr-qc]

[7] T. Damour, A. Nagar, E. N. Dorband, D. Pollney, and L. Rezzolla, Phys.Rev. D77, 084017 (2008) arXiv:0712.3003 [gr-qc].
[8] B. P. Abbott et al. (Virgo, LIGO Scientific), (2016), arXiv:1602.03840 [gr-qc].

[9] B. P. Abbott et al. (Virgo, LIGO Scientific), (2016), arXiv:1602.03841 [gr-qc],

[10] B. P. Abbott et al. (Virgo, LIGO Scientific), Phys. Rev. Lett. 116, 061102 (2016), arXiv:1602.03837 [gr-qc]

[11] B. P. Abbott et al. (Virgo, LIGO Scientific), Phys. Rev. Lett. 116, 241103 (2016), arXiv:1606.04855 [gr-qc]

[12] B. P. Abbott et al. (Virgo, LIGO Scientific), Phys. Rev. X6, 041015 (2016), arXiv:1606.04856 [gr-qc]

[13] S. Khan, S. Husa, M. Hannam, F. Ohme, M. Prrer, X. Jimnez Forteza, and A. Boh, Phys. Rev. D93, 044007 (2016), arXiv:1508.07253 [gr-qc]

[14] Y. Pan, A. Buonanno, A. Taracchini, L. E. Kidder, A. H. Mroué, H. P. Pfeiffer, M. A. Scheel, and B. Szilágyi, 
TABLE II. Fits of the NR quantities extracted at the NQC-extraction point, $t_{\mathrm{NR}}^{\mathrm{NQC}} / M=\left(t_{\mathrm{mrg}}^{\mathrm{nR}}+2\right) / M$ that are used for the iterative determination of the NQC parameters $\left(a_{1}, a_{2}, b_{1}, b_{2}\right)$ for $q=1$.

\begin{tabular}{l}
\hline \hline$A_{22}^{\mathrm{NQC}}=0.00178195 \hat{a}_{0}^{4}+0.00435589 \hat{a}_{0}^{3}+0.00344489 \hat{a}_{0}^{2}-0.00076165 \hat{a}_{0}+0.31973334$ \\
$\dot{A}_{22}^{\mathrm{NQC}}=0.00000927 \hat{a}_{0}^{4}-0.00024550 \hat{a}_{0}^{3}+0.00012469 \hat{a}_{0}^{2}+0.00123845 \hat{a}_{0}-0.00195014$ \\
$\dot{\omega}_{22}^{\mathrm{NQC}}=0.00603482 \hat{a}_{0}^{4}+0.01604555 \hat{a}_{0}^{3}+0.02290799 \hat{a}_{0}^{2}+0.07084587 \hat{a}_{0}+0.38321834$ \\
$\dot{\omega}_{22}^{\mathrm{NQC}}=0.00024066 \hat{a}_{0}^{4}+0.00038123 \hat{a}_{0}^{3}-0.00049714 \hat{a}_{0}^{2}+0.00041219 \hat{a}_{0}+0.01190548$ \\
\hline \hline
\end{tabular}

TABLE III. Same as Table II but for $q>1$ and using the NR dataset discussed in Fig. 3 . In this case, the corresponding $\omega_{22}^{\mathrm{NQC}}$ is given by Eq. A2 above.

\begin{tabular}{lr}
\hline \hline$A_{22}^{\mathrm{NQC}}=$ & $(0.05385059 \nu-0.00890942) \hat{a}_{0}^{2}+(-0.07942102 \nu+0.02152423) \hat{a}_{0}+(0.14805262 \nu+0.28210487)$ \\
$\dot{A}_{22}^{\mathrm{NQC}}=$ & $(0.00248472 \nu-0.00033422) \hat{a}_{0}^{2}+(0.00105298 \nu+0.00085160) \hat{a}_{0}+(-0.00339257 \nu-0.00110932)$ \\
$\dot{\omega}_{22}^{\mathrm{NQC}}=$ & $(-0.00177362 \nu+0.00123900) \hat{a}_{0}+(0.02424739 \nu+0.00566504)$ \\
\hline \hline
\end{tabular}

Phys. Rev. D 89, 084006 (2014).

[15] S. Babak, A. Taracchini, and A. Buonanno, Phys. Rev. D95, 024010 (2017), arXiv:1607.05661 [gr-qc]

[16] B. Abbott et al. (Virgo, LIGO Scientific), Phys. Rev. X6, 041014 (2016), arXiv:1606.01210 [gr-qc]

[17] A. Nagar, T. Damour, C. Reisswig, and D. Pollney, Phys. Rev. D93, 044046 (2016), arXiv:1506.08457 [gr-qc].

[18] S. Balmelli and T. Damour, Phys. Rev. D92, 124022 (2015), arXiv:1509.08135 [gr-qc]

[19] A. Taracchini, A. Buonanno, Y. Pan, T. Hinderer, M. Boyle, et al., Phys.Rev. D89, 061502 (2014), arXiv:1311.2544 [gr-qc].

[20] A. Boh et al., Phys. Rev. D95, 044028 (2017) arXiv:1611.03703 [gr-qc]

[21] D. Bini and T. Damour, Phys.Rev. D87, 121501 (2013), arXiv:1305.4884 [gr-qc].

[22] A. Boh, S. Marsat, and L. Blanchet, Class. Quant. Grav. 30, 135009 (2013), arXiv:1303.7412 [gr-qc]

[23] A. Nagar and A. G. Shah, (2016), arXiv:1606.00207 [grqc]

[24] T. Damour, A. Nagar, and S. Bernuzzi, Phys.Rev. D87, 084035 (2013), arXiv:1212.4357 [gr-qc].

[25] T. Damour, B. R. Iyer, and A. Nagar, Phys. Rev. D79, 064004 (2009)

[26] Y. Pan, A. Buonanno, R. Fujita, E. Racine, and H. Tagoshi, Phys.Rev. D83, 064003 (2011) arXiv:1006.0431 [gr-qc].

[27] G. Faye, L. Blanchet, and B. R. Iyer, Class. Quant. Grav. 32, 045016 (2015), arXiv:1409.3546 [gr-qc]

[28] http://www.black-holes.org/waveforms.

[29] A. Nagar, (2016), arXiv:1606.03952 [gr-qc]

[30] M. A. Scheel et al., Phys. Rev. D79, 024003 (2009)

[31] B. Szilagyi, L. Lindblom, and M. A. Scheel, Phys. Rev. D80, 124010 (2009), arXiv:0909.3557 [gr-qc].

[32] D. A. Hemberger, M. A. Scheel, L. E. Kidder, B. Szilgyi, G. Lovelace, N. W. Taylor, and S. A. Teukolsky, Class. Quant. Grav. 30, 115001 (2013), arXiv:1211.6079 [gr-qc].

[33] L. T. Buchman, H. P. Pfeiffer, M. A. Scheel, and B. Szilagyi, Phys.Rev. D86, 084033 (2012).

[34] D. A. Hemberger, G. Lovelace, T. J. Loredo, L. E. Kidder, M. A. Scheel, et al., Phys.Rev. D88, 064014 (2013), arXiv:1305.5991 [gr-qc].

[35] M. A. Scheel, M. Giesler, D. A. Hemberger, G. Lovelace, K. Kuper, et al., Class.Quant.Grav. 32, 105009 (2015),
arXiv:1412.1803 [gr-qc]

[36] J. Blackman, S. E. Field, C. R. Galley, B. Szilagyi, M. A. Scheel, et al., (2015), arXiv:1502.07758 [gr-qc]

[37] G. Lovelace, M. Boyle, M. A. Scheel, and B. Szilagyi, Class.Quant.Grav. 29, 045003 (2012), arXiv:1110.2229 [gr-qc]

[38] G. Lovelace, M. Scheel, and B. Szilagyi, Phys.Rev. D83, 024010 (2011), arXiv:1010.2777 [gr-qc],

[39] G. Lovelace, M. A. Scheel, R. Owen, M. Giesler, R. Katebi, et al., Class.Quant.Grav. 32, 065007 (2015) arXiv:1411.7297 [gr-qc].

[40] T. Chu, H. Fong, P. Kumar, H. P. Pfeiffer, M. Boyle, D. A. Hemberger, L. E. Kidder, M. A. Scheel, and B. Szilagyi, Class. Quant. Grav. 33, 165001 (2016) arXiv:1512.06800 [gr-qc],

[41] B. Szilgyi, J. Blackman, A. Buonanno, A. Taracchini, H. P. Pfeiffer, M. A. Scheel, T. Chu, L. E. Kidder, and Y. Pan, Phys. Rev. Lett. 115, 031102 (2015), arXiv:1502.04953 [gr-qc].

[42] G. Lovelace et al., Class. Quant. Grav. 33, 244002 (2016) arXiv:1607.05377 [gr-qc].

[43] B. P. Abbott et al. (Virgo, LIGO Scientific), (2016), arXiv:1606.01262 [gr-qc].

[44] B. Bruegmann, J. A. Gonzalez, M. Hannam, S. Husa, U. Sperhake, and W. Tichy, Phys. Rev. D77, 024027 (2008), arXiv:gr-qc/0610128 [gr-qc].

[45] S. Husa, J. A. Gonzalez, M. Hannam, B. Bruegmann, and U. Sperhake, Class. Quant. Grav. 25, 105006 (2008), arXiv:0706.0740 [gr-qc].

[46] J. A. Gonzalez et al., Phys. Rev. Lett. 98, 091101 (2007)

[47] A. H. Mroue, M. A. Scheel, B. Szilagyi, H. P. Pfeiffer, M. Boyle, et al., Phys.Rev.Lett. 111, 241104 (2013), arXiv:1304.6077 [gr-qc].

[48] S. Husa, S. Khan, M. Hannam, M. Prrer, F. Ohme, X. J. Forteza, and A. Boh, (2015), arXiv:1508.07250 [gr-qc].

[49] A. Nagar, Phys.Rev. D84, 084028 (2011) arXiv:1106.4349 [gr-qc].

[50] E. Barausse and A. Buonanno, Phys.Rev. D84, 104027 (2011), arXiv:1107.2904 [gr-qc]

[51] T. Damour and A. Nagar, Phys.Rev. D90, 044018 (2014), arXiv:1406.6913 [gr-qc]

[52] T. Damour and A. Nagar, Phys. Rev. D90, 024054 (2014), arXiv:1406.0401 [gr-qc] 
TABLE IV. Same as Table II but for $q>1$ and using the NR dataset discussed in Fig. 11. In this case, the corresponding $\omega_{22}^{\mathrm{NQC}^{\prime}}$ is given by Eq. A3 above.

\begin{tabular}{ll}
\hline \hline$A_{22}^{\mathrm{NQC}^{\prime}}=$ & $(0.04680896 \nu-0.00632114) \hat{a}_{0}^{3}+(0.06586192 \nu-0.01180039) \hat{a}_{0}^{2}+(-0.11617413 \nu+0.02704959) \hat{a}_{0}$ \\
& $+(0.15597465 \nu+0.28034978)$ \\
$\dot{A}_{22}^{\mathrm{NQC}^{\prime}}=$ & $(-0.00130824 \nu+0.00006202) \hat{a}_{0}^{3}+(0.00199855 \nu-0.00027474) \hat{a}_{0}^{2}+(0.00218838 \nu+0.00071540) \hat{a}_{0}$ \\
& $+(-0.00362779 \nu-0.00105397)$ \\
$\dot{\omega}_{22}^{\mathrm{NQC}}=$ & $(0.00061175 \nu+0.00074001) \hat{a}_{0}+(0.02504442 \nu+0.00548217)$ \\
\hline
\end{tabular}

TABLE V. Nonspinning NR datasets considered in this work. Datesets form 1 to 9 were used in the determination of $a_{5}^{c}$ in Paper I and the related fits of the NQC parameters. The others are used here just for validation.

\begin{tabular}{|c|c|c|c|c|c|c|c|}
\hline$\#$ & Name & $\mathrm{N}$ orbits & $\nu$ & $q$ & $\delta \phi_{\mathrm{mrg}}^{\mathrm{NR}}[\mathrm{rad}]$ & $\Delta \phi_{\mathrm{mrg}}^{\mathrm{EOBNR}}[\mathrm{rad}]$ & $\max (\bar{F})[\%]$ \\
\hline 1 & SXS:BBH:0066 & 28.1 & 0.25 & 1.00 & $\ldots$ & +0.0599 & 0.10 \\
\hline 2 & SXS:BBH:0002 & 32.4 & 0.25 & 1.00 & -0.0633 & +0.1630 & 0.47 \\
\hline 3 & SXS:BBH:0007 & 29.1 & 0.24 & 1.50 & -0.0183 & +0.0560 & 0.07 \\
\hline 4 & SXS:BBH:0169 & 15.7 & 0.22 & 2.00 & -0.0267 & -0.1537 & 0.05 \\
\hline 5 & SXS:BBH:0030 & 18.2 & 0.19 & 3.00 & -0.0857 & -0.0733 & 0.04 \\
\hline 6 & SXS:BBH:0167 & 15.6 & 0.16 & 4.00 & -0.5076 & -0.0585 & 0.04 \\
\hline 7 & SXS:BBH:0056 & 28.8 & 0.14 & 5.00 & +0.4405 & -0.0892 & 0.03 \\
\hline 8 & SXS:BBH:0166 & 21.6 & 0.12 & 6.00 & $\ldots$ & -0.2163 & 0.03 \\
\hline 9 & SXS:BBH:0063 & 25.8 & 0.10 & 8.00 & +1.0118 & -0.4310 & 0.04 \\
\hline 10 & SXS:BBH:0185 & 24.9 & 0.08 & 9.99 & +0.3499 & -0.5839 & 0.07 \\
\hline 11 & SXS:BBH:0180 & 28.2 & 0.25 & 1.00 & -0.4201 & +0.0709 & 0.11 \\
\hline 12 & SXS:BBH:0259 & 28.6 & 0.20 & 2.50 & -0.0081 & +0.0403 & 0.02 \\
\hline 13 & SXS:BBH:0295 & 27.8 & 0.15 & 4.50 & +0.2413 & -0.0254 & 0.03 \\
\hline 14 & SXS:BBH:0296 & 27.9 & 0.13 & 5.50 & +0.4442 & -0.1595 & 0.02 \\
\hline 15 & SXS:BBH:0297 & 19.7 & 0.12 & 6.50 & -0.0471 & -0.2669 & 0.03 \\
\hline 16 & SXS:BBH:0298 & 19.7 & 0.11 & 7.00 & -0.0690 & -0.2874 & 0.03 \\
\hline 17 & SXS:BBH:0299 & 20.1 & 0.10 & 7.50 & -0.0410 & -0.3097 & 0.04 \\
\hline 18 & SXS:BBH:0300 & 18.7 & 0.09 & 8.50 & -0.0671 & -0.4308 & 0.05 \\
\hline 19 & SXS:BBH:0301 & 18.9 & 0.09 & 9.00 & -0.1509 & -0.3616 & 0.03 \\
\hline 20 & SXS:BBH:0302 & 19.1 & 0.09 & 9.50 & +0.0318 & -0.3947 & 0.03 \\
\hline 21 & SXS:BBH:0303 & 19.3 & 0.08 & 10.00 & +0.3037 & -0.4566 & 0.03 \\
\hline
\end{tabular}

[53] J. Healy, C. O. Lousto, and Y. Zlochower, Phys. Rev. D90, 104004 (2014), arXiv:1406.7295 [gr-qc]

[54] E. Berti, V. Cardoso, and C. M. Will, Phys.Rev. D73, 064030 (2006), arXiv:gr-qc/0512160 [gr-qc]

[55] E. Berti, http://www.phy.olemiss.edu/berti/qnms.html.

[56] D. Shoemaker,

[57] A. Nagar and L. Rezzolla, Class.Quant.Grav. 22, R167 (2005)

[58] L. Baiotti, T. Damour, B. Giacomazzo, A. Nagar, and L. Rezzolla, Phys.Rev. D84, 024017 (2011), arXiv:1103.3874 [gr-qc]

[59] Y. Pan, A. Buonanno, M. Boyle, L. T. Buchman, L. E. Kidder, et al., Phys.Rev. D84, 124052 (2011), arXiv:1106.1021 [gr-qc]].

[60] T. Damour, B. R. Iyer, and B. S. Sathyaprakash, Phys. Rev. D57, 885 (1998), arXiv:gr-qc/9708034 [gr-qc]]
[61] A. Taracchini, Y. Pan, A. Buonanno, E. Barausse, M. Boyle, et al., Phys.Rev. D86, 024011 (2012), arXiv:1202.0790 [gr-qc].

[62] Y. Pan, A. Buonanno, L. T. Buchman, T. Chu, L. E. Kidder, et al., Phys.Rev. D81, 084041 (2010), arXiv:0912.3466 [gr-qc].

[63] T. Damour and A. Nagar, Phys. Rev. D79, 081503 (2009)

[64] M. Boyle, (2015), arXiv:1509.00862 [gr-qc]

[65] S. Ossokine, F. Foucart, H. P. Pfeiffer, M. Boyle, and B. Szilgyi, (2015), arXiv:1506.01689 [gr-qc]

[66] I. Kamaretsos, M. Hannam, S. Husa, and B. S. Sathyaprakash, Phys. Rev. D85, 024018 (2012), arXiv:1107.0854 [gr-qc],

[67] I. Kamaretsos, M. Hannam, and B. Sathyaprakash, Phys. Rev. Lett. 109, 141102 (2012), arXiv:1207.0399 [gr-qc] 
TABLE VI. Spinning binaries: original spin-aligned SXS datasets used in Paper I. The columns report: the number of the dataset; the name of the configuration on the SXS catalog; the symmetric mass ratio $\nu ;\left(q, \chi_{1}, \chi_{2}\right)$, where $q=m_{1} / m_{2}$ is the mass ratio and $\chi_{1,2}$ are the dimensionless spins; the NR phase uncertainty at merger (when available), in radians, measured taking the difference between the two highest resolution levels (see text for details); the EOB/NR phase difference, in radians, at merger computed using EOB models informed with increasing amount of NR information (see text for details) and imposing an EOB/NR alignment during the early inspiral; the corresponding maximal unfaithfulnesses $\max (\bar{F})$, see Eq. (14).

\begin{tabular}{|c|c|c|c|c|c|c|c|c|c|c|c|}
\hline \multirow[t]{2}{*}{$\#$} & \multirow[t]{2}{*}{ Name } & \multirow[t]{2}{*}{$\mathrm{N}$ orbits } & \multirow[t]{2}{*}{$\nu$} & \multirow[t]{2}{*}{$\left(q, \chi_{1}, \chi_{2}\right)$} & \multirow[t]{2}{*}{$\delta \phi_{\mathrm{mrg}}^{\mathrm{NR}}$} & \multicolumn{3}{|c|}{$\Delta \phi_{\mathrm{mrg}}^{\mathrm{EOBNR}}$} & \multicolumn{3}{|c|}{$\max (\bar{F})[\%]$} \\
\hline & & & & & & $c_{3}^{\text {old }_{\mathrm{NQC}}}$ & $c_{3}^{\text {new NQC }}$ & $c_{3}^{\text {new }{ }^{N Q C^{\prime}}}$ & $c_{3}^{\text {old } \mathrm{NQC}}$ & $c_{3}^{\text {new NQC }}$ & $c_{3}^{\text {new }_{\mathrm{NQC}}}$ \\
\hline 22 & SXS:BBH:0004 & 30.2 & 0.25 & $(1,-0.50,0)$ & +0.0680 & +0.3731 & +0.7742 & +0.7742 & 0.07 & 0.09 & 0.09 \\
\hline 23 & SXS:BBH:0005 & 30.2 & 0.25 & $(1,+0.50,0)$ & +0.2791 & +0.2930 & +0.8445 & +0.8445 & 0.04 & 0.15 & 0.15 \\
\hline 24 & SXS:BBH:0156 & 12.4 & 0.25 & $(1,-0.95,-0.95)$ & -2.0991 & +0.3389 & +0.3389 & +0.3389 & 0.08 & 0.08 & 0.08 \\
\hline 25 & SXS:BBH:0159 & 12.7 & 0.25 & $(1,-0.90,-0.90)$ & +0.3778 & +0.0746 & +0.0746 & +0.0746 & 0.08 & 0.08 & 0.08 \\
\hline 26 & SXS:BBH:0154 & 13.2 & 0.25 & $(1,-0.80,-0.80)$ & -0.0058 & +0.1516 & +0.1516 & +0.1516 & 0.08 & 0.08 & 0.08 \\
\hline 27 & SXS:BBH:0151 & 14.5 & 0.25 & $(1,-0.60,-0.60)$ & +0.1347 & -0.0082 & -0.0082 & -0.0082 & 0.05 & 0.05 & 0.05 \\
\hline 28 & SXS:BBH:0148 & 15.5 & 0.25 & $(1,-0.44,-0.44)$ & +0.6981 & +0.8424 & +0.8424 & +0.8424 & 0.10 & 0.10 & 0.10 \\
\hline 29 & SXS:BBH:0149 & 17.1 & 0.25 & $(1,-0.20,-0.20)$ & -0.9015 & +0.5001 & +0.5001 & +0.5001 & 0.14 & 0.14 & 0.14 \\
\hline 30 & SXS:BBH:0150 & 19.8 & 0.25 & $(1,+0.20,+0.20)$ & -0.9593 & +1.0733 & +1.0733 & +1.0733 & 0.25 & 0.25 & 0.25 \\
\hline 31 & SXS:BBH:0152 & 22.6 & 0.25 & $(1,+0.60,+0.60)$ & +0.3577 & -0.0211 & -0.0211 & -0.0211 & 0.05 & 0.05 & 0.05 \\
\hline 32 & SXS:BBH:0155 & 24.1 & 0.25 & $(1,+0.80,+0.80)$ & +0.2657 & -0.3884 & -0.3884 & -0.3884 & 0.24 & 0.24 & 0.24 \\
\hline 33 & SXS:BBH:0153 & 24.5 & 0.25 & $(1,+0.85,+0.85)$ & & +0.1635 & +0.1635 & +0.1635 & 0.24 & 0.24 & 0.24 \\
\hline 34 & SXS:BBH:0160 & 24.8 & 0.25 & $(1,+0.90,+0.90)$ & +0.7893 & +0.2250 & +0.2250 & +0.2250 & 0.46 & 0.46 & 0.46 \\
\hline 35 & SXS:BBH:0157 & 25.2 & 0.25 & $(1,+0.95,+0.95)$ & +1.1689 & +0.2341 & +0.2341 & +0.2341 & 0.77 & 0.77 & 0.77 \\
\hline 36 & SXS:BBH:0158 & 25.3 & 0.25 & $(1,+0.97,+0.97)$ & +1.2450 & +0.8158 & +0.8158 & +0.8158 & 0.69 & 0.69 & 0.69 \\
\hline 37 & SXS:BBH:0172 & 25.4 & 0.25 & $(1,+0.98,+0.98)$ & +1.9682 & +0.4869 & +0.4869 & +0.4869 & 0.78 & 0.78 & 0.78 \\
\hline 38 & SXS:BBH:0177 & 25.4 & 0.25 & $(1,+0.99,+0.99)$ & +0.3929 & +0.3418 & +0.3418 & +0.3418 & 0.83 & 0.83 & 0.83 \\
\hline 39 & SXS:BBH:0178 & 25.4 & 0.25 & $(1,+0.994,+0.994)$ & -0.5200 & +0.3370 & +0.3370 & +0.3370 & 0.95 & 0.95 & 0.95 \\
\hline 40 & SXS:BBH:0013 & 23.8 & 0.24 & $(1.50,+0.50,0)$ & & +0.6178 & +0.8734 & +0.8450 & 0.14 & 0.20 & 0.19 \\
\hline 41 & SXS:BBH:0014 & 22.6 & 0.24 & $(1.50,-0.50,0)$ & +0.1450 & +0.1839 & +0.3484 & +0.3413 & 0.04 & 0.04 & 0.04 \\
\hline 42 & SXS:BBH:0162 & 18.6 & 0.22 & $(2.00,+0.60,0)$ & -0.6708 & -0.3531 & -0.1951 & -0.2199 & 0.05 & 0.12 & 0.11 \\
\hline 43 & SXS:BBH:0036 & 31.7 & 0.19 & $(3.00,-0.50,0)$ & -0.0642 & +0.8544 & +0.9466 & +0.9408 & 0.03 & 0.03 & 0.03 \\
\hline 44 & SXS:BBH:0031 & 21.9 & 0.19 & $(3.00,+0.50,0)$ & +0.0346 & +0.1774 & +0.3294 & +0.3288 & 0.05 & 0.06 & 0.06 \\
\hline 45 & SXS:BBH:0047 & 22.7 & 0.19 & $(3.00,+0.50,+0.50)$ & $\ldots$ & +0.0811 & -0.1858 & -0.2240 & 0.06 & 0.15 & 0.09 \\
\hline 46 & SXS:BBH:0046 & 14.4 & 0.19 & $(3.00,-0.50,-0.50)$ & & +0.4618 & +0.3490 & +0.3434 & 0.08 & 0.05 & 0.07 \\
\hline 47 & SXS:BBH:0110 & 24.2 & 0.14 & $(5.00,+0.50,0)$ & & +0.0832 & +0.2869 & +0.2594 & 0.03 & 0.04 & 0.05 \\
\hline 48 & SXS:BBH:0060 & 23.2 & 0.14 & $(5.00,-0.50,0)$ & $\cdots$ & +0.5855 & +0.7012 & +0.6994 & 0.04 & 0.04 & 0.04 \\
\hline 49 & SXS:BBH:0064 & 19.2 & 0.10 & $(8.00,-0.50,0)$ & +0.8034 & +0.0499 & +0.0286 & +0.0001 & 0.06 & 0.06 & 0.06 \\
\hline 50 & SXS:BBH:0065 & 34.0 & 0.10 & $(8.00,+0.50,0)$ & -2.9703 & +0.9736 & +0.8511 & +0.7970 & 0.02 & 0.03 & 0.04 \\
\hline
\end{tabular}

[68] A. Taracchini, A. Buonanno, G. Khanna, and S. A. Hughes, (2014), arXiv:1404.1819 [gr-qc].

[69] E. Harms, S. Bernuzzi, A. Nagar, and A. Zenginoglu, (2014), arXiv:1406.5983 [gr-qc] 
TABLE VII. Same structure of TableVI, but referring to new spin-aligned configurations, with $q<2$, not used in Paper I. The datasets in bold, that give the largest maximal unfaithfulness when the $c_{3}^{\text {old }_{\mathrm{NQC}}}$ determination is used, are the only ones used to provide the improved determination of $c_{3}$ that yield the $c_{3}^{\text {new }}{ }^{N_{\mathrm{NC}}}$ and $c_{3}{ }_{\text {new } \mathrm{NQ}^{\prime}}$ values for $\Delta \phi_{\operatorname{mrg}}^{\mathrm{EOBNR}}$ and $\max (\bar{F})$.

\begin{tabular}{|c|c|c|c|c|c|c|c|c|c|c|c|}
\hline \multirow[t]{2}{*}{$\#$} & \multirow[t]{2}{*}{ Name } & \multirow[t]{2}{*}{$\mathrm{N}$ orbits } & & \multirow[t]{2}{*}{$\left(q, \chi_{1}, \chi_{2}\right)$} & \multirow[t]{2}{*}{$\delta \phi_{\mathrm{NR}}^{\mathrm{mrg}}$} & \multicolumn{3}{|c|}{$\Delta \phi_{\mathrm{mrg}}^{\mathrm{EOBNR}}$} & \multicolumn{3}{|c|}{$\max (\bar{F})[\%]$} \\
\hline & & & & & & $c_{3}^{\text {old }_{\mathrm{NQC}}}$ & $c_{3}^{\text {newNQC }}$ & $c_{3}^{\text {new }}{ }^{N_{Q C}{ }^{\prime}}$ & $c_{3}^{\text {old }_{\mathrm{NQC}}}$ & $c_{3}^{\text {new NQC }}$ & $c_{3}^{\text {new }}{ }^{\mathrm{NQC}^{\prime}}$ \\
\hline 51 & SXS:BBH:0212 & 28.6 & 0.25 & $(1,-0.80,-0.80)$ & -3.1754 & +1.6613 & +1.6613 & +1.6613 & 0.08 & 0.08 & 0.08 \\
\hline 52 & SXS:BBH:0215 & 25.8 & 0.25 & $(1,-0.60,-0.60)$ & +2.1069 & +0.9068 & +0.9068 & +0.9068 & 0.04 & 0.04 & 0.04 \\
\hline 53 & SXS:BBH:0170 & 15.5 & 0.25 & $(1,+0.44,+0.44)$ & +0.0278 & -0.4039 & -0.4039 & -0.4039 & 0.07 & 0.08 & 0.08 \\
\hline 54 & SXS:BBH:0228 & 23.5 & 0.25 & $(1,+0.60,+0.60)$ & +2.4480 & +0.0888 & +0.0888 & +0.0888 & 0.05 & 0.05 & 0.05 \\
\hline 55 & SXS:BBH:0230 & 24.2 & 0.25 & $(1,+0.80,+0.80)$ & -1.3471 & -0.2300 & -0.2300 & -0.2300 & 0.25 & 0.25 & 0.25 \\
\hline 56 & SXS:BBH:0223 & 23.3 & 0.25 & $(1,+0.30,+0.00)$ & +0.1170 & +0.9346 & +1.1444 & +1.1444 & 0.12 & 0.21 & 0.21 \\
\hline 57 & SXS:BBH:0222 & 23.6 & 0.25 & $(1,-0.30,+0.00)$ & +0.1361 & +0.6240 & +0.7713 & +0.7713 & 0.05 & 0.06 & 0.06 \\
\hline 58 & SXS:BBH:0225 & 23.5 & 0.25 & $(1,+0.40,+0.80)$ & +2.5723 & +2.2891 & +0.9983 & +0.9983 & 0.47 & 0.09 & 0.09 \\
\hline 59 & SXS:BBH:0224 & 22.9 & 0.25 & $(1,+0.40,-0.80)$ & +1.8737 & +2.2534 & +1.3953 & +1.3953 & 0.29 & 0.17 & 0.17 \\
\hline 60 & SXS:BBH:0221 & 22.7 & 0.25 & $(1,-0.40,+0.80)$ & +1.7979 & +3.0846 & +1.9880 & +1.9880 & 0.80 & 0.44 & 0.44 \\
\hline 61 & SXS:BBH:0220 & 25.7 & 0.25 & $(1,-0.40,-0.80)$ & +1.1940 & +1.9703 & +1.3057 & +1.3057 & 0.13 & 0.09 & 0.09 \\
\hline 62 & SXS:BBH:0226 & 22.9 & 0.25 & $(1,+0.50,-0.90)$ & +2.4759 & +2.5333 & +1.4938 & +1.4938 & 0.34 & 0.17 & 0.17 \\
\hline 63 & SXS:BBH:0219 & 22.4 & 0.25 & $(1,-0.50,+0.90)$ & +1.0612 & +3.4057 & +2.1497 & +2.1497 & 0.93 & 0.48 & 0.48 \\
\hline 64 & SXS:BBH:0218 & 29.1 & 0.25 & $(1,-0.50,+0.50)$ & +0.4240 & +1.5324 & +1.5324 & +1.5324 & 0.17 & 0.17 & 0.17 \\
\hline 65 & SXS:BBH:0227 & 23.1 & 0.25 & $(1,+0.60,+0.00)$ & +2.3492 & -0.1544 & +0.6109 & +0.6109 & 0.04 & 0.12 & 0.12 \\
\hline 66 & SXS:BBH:0217 & 22.7 & 0.25 & $(1,-0.60,+0.60)$ & +1.6009 & +1.1389 & +1.1389 & +1.1389 & 0.19 & 0.19 & 0.19 \\
\hline 67 & SXS:BBH:0216 & 23.6 & 0.25 & $(1,-0.60,+0.00)$ & +2.2546 & -0.0606 & +0.3930 & +0.3930 & 0.07 & 0.05 & 0.05 \\
\hline 68 & SXS:BBH:0214 & 24.4 & 0.25 & $(1,-0.62,-0.25)$ & +1.8662 & -0.9374 & -0.6141 & -0.6141 & 0.11 & 0.08 & 0.08 \\
\hline 69 & SXS:BBH:0229 & 23.1 & 0.25 & $(1,+0.65,+0.25)$ & +2.3005 & -0.6130 & +0.1326 & +0.1326 & 0.10 & 0.04 & 0.04 \\
\hline 70 & SXS:BBH:0213 & 22.3 & 0.25 & $(1,-0.80,+0.80)$ & +1.2991 & +1.0759 & +1.0760 & +1.0760 & 0.20 & 0.20 & 0.20 \\
\hline 71 & SXS:BBH:0232 & 23.9 & 0.25 & $(1,+0.90,+0.50)$ & +1.7528 & -2.4962 & -1.3867 & -1.3867 & 2.49 & 0.89 & 0.89 \\
\hline 72 & SXS:BBH:0231 & 23.1 & 0.25 & $(1,+0.90,+0.00)$ & +2.8942 & -2.1568 & -0.7340 & -0.7340 & 1.39 & 0.20 & 0.20 \\
\hline 73 & SXS:BBH:0211 & 22.3 & 0.25 & $(1,-0.90,+0.90)$ & +2.2000 & +1.0183 & +1.0301 & +1.0301 & 0.14 & 0.21 & 0.21 \\
\hline 74 & SXS:BBH:0210 & 24.3 & 0.25 & $(1,-0.90,+0.00)$ & +1.1308 & -0.8632 & -0.0140 & -0.0140 & 0.22 & 0.07 & 0.07 \\
\hline 75 & SXS:BBH:0209 & 27.0 & 0.25 & $(1,-0.90,-0.50)$ & -2.2779 & +0.0777 & +0.6918 & +0.6918 & 0.11 & 0.07 & 0.07 \\
\hline 76 & SXS:BBH:0306 & 12.6 & 0.25 & $(1.31,+0.96,-0.90)$ & +0.7815 & -1.2673 & -0.3053 & -0.2937 & 0.29 & 0.40 & 0.39 \\
\hline 77 & SXS:BBH:0025 & 22.4 & 0.24 & $(1.50,+0.50,-0.50)$ & +0.1348 & +0.7431 & +1.1521 & +1.1795 & 0.20 & 0.28 & 0.39 \\
\hline 78 & SXS:BBH:0019 & 20.4 & 0.24 & $(1.50,-0.50,+0.50)$ & -0.3626 & -0.1380 & +0.1427 & +0.1416 & 0.05 & 0.04 & 0.04 \\
\hline 79 & SXS:BBH:0016 & 30.7 & 0.24 & $(1.50,-0.50,+0.00)$ & -0.0311 & +0.6344 & +0.8033 & +0.7960 & 0.03 & 0.03 & 0.03 \\
\hline
\end{tabular}


TABLE VIII. Same as TableVII but referring to $q=2$, spin-aligned, BBH configurations. The dataset in bold, SXS:BBH:0257, is the only one used of this set to provide the new determination of $c_{3}$ that yields the $c_{3}^{\text {new }_{\mathrm{NQC}}}$ values for $\Delta \phi_{\mathrm{mrg}}^{\mathrm{EOBN}}$ and max $(\bar{F})$.

\begin{tabular}{|c|c|c|c|c|c|c|c|c|c|c|c|}
\hline \multirow[t]{2}{*}{$\#$} & \multirow[t]{2}{*}{ Name } & \multirow[t]{2}{*}{$\mathrm{N}$ orbits } & \multirow[t]{2}{*}{$\nu$} & \multirow[t]{2}{*}{$\left(q, \chi_{1}, \chi_{2}\right)$} & \multirow[t]{2}{*}{$\delta \phi_{\mathrm{NR}}^{\mathrm{mrg}}$} & \multicolumn{3}{|c|}{$\Delta \phi_{\mathrm{mrg}}^{\mathrm{EOBNR}}$} & \multicolumn{3}{|c|}{$\max (\bar{F})[\%]$} \\
\hline & & & & & & $c_{3}^{\text {old }_{\mathrm{NQC}}}$ & $c_{3}^{\text {new }}$ & $c_{3}^{\text {new } \mathrm{NQC}^{\prime}}$ & $c_{3}^{\text {old }_{\mathrm{NQC}}}$ & $c_{3}^{\text {new }}$ & $c_{3}^{c^{\text {new }} \mathrm{NQC}^{\prime}}$ \\
\hline 80 & SXS:BBH:0234 & 27.8 & 0.22 & $(2,-0.85,-0.85)$ & -0.5179 & +3.3569 & +1.9117 & +1.9039 & 0.21 & 0.13 & 0.13 \\
\hline 81 & SXS:BBH:0235 & 25.1 & 0.22 & $(2,-0.60,-0.60)$ & +0.7081 & +1.8290 & +1.1071 & +1.0990 & 0.11 & 0.08 & 0.08 \\
\hline 82 & SXS:BBH:0238 & 32.0 & 0.22 & $(2,-0.50,-0.50)$ & +0.2792 & +2.2538 & +1.6376 & +1.6295 & 0.09 & 0.06 & 0.06 \\
\hline 83 & SXS:BBH:0240 & 23.5 & 0.22 & $(2,-0.30,-0.30)$ & +0.0472 & +1.0541 & +0.8448 & +0.8386 & 0.06 & 0.05 & 0.05 \\
\hline 84 & SXS:BBH:0243 & 23.3 & 0.22 & $(2,-0.13,-0.85)$ & +2.0139 & +2.7099 & +1.8501 & +1.8432 & 0.32 & 0.21 & 0.22 \\
\hline 85 & SXS:BBH:0248 & 23.2 & 0.22 & $(2,+0.13,+0.85)$ & +0.9247 & +2.4351 & +1.3149 & +1.3294 & 0.36 & 0.23 & 0.23 \\
\hline 86 & SXS:BBH:0251 & 23.5 & 0.22 & $(2,+0.30,+0.30)$ & +0.0170 & +1.3405 & +1.0557 & +1.0214 & 0.14 & 0.09 & 0.08 \\
\hline 87 & SXS:BBH:0253 & 28.8 & 0.22 & $(2,+0.50,+0.50)$ & +0.1010 & +1.4502 & +0.4482 & +0.4217 & 0.15 & 0.06 & 0.06 \\
\hline 88 & SXS:BBH:0256 & 23.9 & 0.22 & $(2,+0.60,+0.60)$ & +0.4916 & +1.6769 & +0.1673 & +0.1489 & 0.16 & 0.10 & 0.11 \\
\hline 89 & SXS:BBH:0257 & 24.8 & 0.22 & $(2,+0.85,+0.85)$ & +0.3056 & +3.7223 & +0.4311 & +0.5929 & 1.04 & 0.08 & 0.07 \\
\hline 90 & SXS:BBH:0247 & 22.6 & 0.22 & $(2,+0.00,+0.60)$ & +1.4523 & +1.3997 & +1.0516 & +1.0256 & 0.12 & 0.11 & 0.16 \\
\hline 91 & SXS:BBH:0246 & 22.9 & 0.22 & $(2,+0.00,+0.30)$ & -4.2316 & +4.2002 & +4.0612 & +4.0646 & 0.07 & 0.06 & 0.06 \\
\hline 92 & SXS:BBH:0245 & 23.0 & 0.22 & $(2,+0.00,-0.30)$ & +0.0053 & +1.2993 & +1.2268 & +1.2112 & 0.12 & 0.11 & 0.07 \\
\hline 93 & SXS:BBH:0244 & 23.2 & 0.22 & $(2,+0.00,-0.60)$ & +1.7648 & +1.9083 & +1.5937 & +1.5893 & 0.23 & 0.19 & 0.19 \\
\hline 94 & SXS:BBH:0250 & 23.2 & 0.22 & $(2,+0.30,+0.00)$ & +0.0114 & +1.0907 & +1.1286 & +1.1359 & 0.13 & 0.13 & 0.13 \\
\hline 95 & SXS:BBH:0249 & 23.2 & 0.22 & $(2,+0.30,-0.30)$ & +0.0207 & +1.0894 & +1.3016 & +1.2862 & 0.13 & 0.23 & 0.16 \\
\hline 96 & SXS:BBH:0242 & 23.1 & 0.22 & $(2,-0.30,+0.30)$ & +0.0381 & +0.2622 & +0.4012 & +0.3991 & 0.04 & 0.04 & 0.04 \\
\hline 97 & SXS:BBH:0241 & 23.1 & 0.22 & $(2,-0.30,+0.00)$ & +0.0372 & +0.5318 & +0.5254 & +0.5607 & 0.03 & 0.03 & 0.03 \\
\hline 98 & SXS:BBH:0252 & 22.5 & 0.22 & $(2,+0.37,-0.85)$ & -2.5921 & +1.6158 & +1.6861 & +1.6855 & 0.48 & 0.51 & 0.51 \\
\hline 99 & SXS:BBH:0239 & 22.2 & 0.22 & $(2,-0.37,+0.85)$ & -2.8781 & -0.1778 & -0.1285 & -0.1272 & 0.11 & 0.11 & 0.11 \\
\hline 100 & SXS:BBH:0255 & 23.3 & 0.22 & $(2,+0.60,+0.00)$ & -0.2908 & +0.0670 & +0.1870 & +0.2071 & 0.05 & 0.12 & 0.11 \\
\hline 101 & SXS:BBH:0254 & 22.9 & 0.22 & $(2,+0.60,-0.60)$ & -0.0309 & +0.0725 & +0.8745 & +0.8826 & 0.44 & 0.61 & 0.60 \\
\hline 102 & SXS:BBH:0237 & 22.6 & 0.22 & $(2,-0.60,+0.60)$ & -1.3705 & -0.8173 & -0.4980 & -0.4676 & 0.17 & 0.09 & 0.09 \\
\hline 103 & SXS:BBH:0236 & 23.4 & 0.22 & $(2,-0.60,+0.00)$ & -0.2104 & -0.2146 & -0.1289 & -0.1348 & 0.06 & 0.05 & 0.06 \\
\hline 104 & SXS:BBH:0258 & 22.8 & 0.22 & $(2,+0.87,-0.85)$ & +0.7293 & -1.8064 & -0.5120 & -0.4985 & 0.98 & 1.49 & 0.43 \\
\hline 105 & SXS:BBH:0233 & 22.0 & 0.22 & $(2,-0.87,+0.85)$ & -1.4586 & -1.8336 & -1.3124 & -1.3166 & 0.54 & 0.29 & 0.31 \\
\hline
\end{tabular}


TABLE IX. Same as Table VIII but referring to spin-algined BBHs with $3 \leq q \leq 7$. We stress that none of the datasets listed here was used in the determination of any value of $c_{3}$, but rather they only served to validate the model.

\begin{tabular}{|c|c|c|c|c|c|c|c|c|c|c|c|}
\hline \multirow[t]{2}{*}{$\#$} & \multirow[t]{2}{*}{ Name } & \multirow[t]{2}{*}{$\mathrm{N}$ orbits } & \multirow[t]{2}{*}{$\nu$} & \multirow[t]{2}{*}{$\left(q, \chi_{1}, \chi_{2}\right)$} & \multirow[t]{2}{*}{$\delta \phi_{\mathrm{NR}}^{\mathrm{mrg}}$} & \multicolumn{3}{|c|}{$\Delta \phi_{\mathrm{mrg}}^{\mathrm{EOBNR}}$} & \multicolumn{3}{|c|}{$\max (\bar{F})[\%]$} \\
\hline & & & & & & $c_{3}^{\text {old }_{\mathrm{NQC}}}$ & & $c_{3}^{\text {new }_{\mathrm{NQC}^{\prime}}}$ & $c_{3}^{\text {old }_{\mathrm{NQC}}}$ & $c_{3}^{\text {new }}{ }^{\text {nC }}$ & $c_{3}^{{ }^{n e w}{ }^{N_{Q} C^{\prime}}}$ \\
\hline 106 & SXS:BBH:0260 & 25.8 & 0.19 & $(3,-0.85,-0.85)$ & -2.8231 & +1.3837 & +1.0296 & +0.9924 & 0.16 & 0.14 & 0.14 \\
\hline 107 & SXS:BBH:0264 & 23.4 & 0.19 & $(3,-0.60,-0.60)$ & -1.9449 & +0.8182 & +0.6367 & +0.6341 & 0.09 & 0.08 & 0.08 \\
\hline 108 & SXS:BBH:0267 & 23.4 & 0.19 & $(3,-0.50,-0.50)$ & -0.1123 & +1.4304 & +1.2883 & +1.2977 & 0.07 & 0.04 & 0.07 \\
\hline 109 & SXS:BBH:0270 & 22.8 & 0.19 & $(3,-0.30,-0.30)$ & +0.0119 & +0.7593 & +0.6971 & +0.6930 & 0.05 & 0.04 & 0.04 \\
\hline 110 & SXS:BBH:0283 & 3.5 & 0.19 & $(3,+0.30,+0.30)$ & -0.0063 & +0.9727 & +0.8730 & +0.8708 & 0.04 & 0.03 & 0.03 \\
\hline 111 & SXS:BBH:0286 & 24.1 & 0.19 & $(3,+0.50,+0.50)$ & -0.3122 & +0.5992 & +0.2557 & +0.2143 & 0.06 & 0.15 & 0.09 \\
\hline 112 & SXS:BBH:0291 & 24.5 & 0.19 & $(3,+0.60,+0.60)$ & +1.3163 & -0.1624 & -0.5959 & -0.6302 & 0.14 & 0.32 & 0.34 \\
\hline 113 & SXS:BBH:0293 & 25.6 & 0.19 & $(3,+0.85,+0.85)$ & +1.1508 & +0.0013 & -0.2404 & -0.1945 & 0.20 & 0.29 & 0.27 \\
\hline 114 & SXS:BBH:0278 & 22.8 & 0.19 & $(3,+0.00,+0.60)$ & +1.8831 & +1.0782 & +0.9450 & +0.9411 & 0.10 & 0.10 & 0.10 \\
\hline 115 & SXS:BBH:0277 & 22.9 & 0.19 & $(3,+0.00,+0.30)$ & +0.0142 & +0.8898 & +0.8964 & +0.8525 & 0.05 & 0.05 & 0.04 \\
\hline 116 & SXS:BBH:0276 & 23.0 & 0.19 & $(3,+0.00,-0.30)$ & -0.0680 & +1.2736 & +1.2354 & +1.2310 & 0.10 & 0.10 & 0.10 \\
\hline 117 & SXS:BBH:0275 & 22.6 & 0.19 & $(3,+0.00,-0.60)$ & -0.8362 & +1.7759 & +1.6612 & +1.6568 & 0.18 & 0.16 & 0.16 \\
\hline 118 & SXS:BBH:0279 & 22.6 & 0.19 & $(3,+0.23,-0.85)$ & -0.2303 & +1.8845 & +1.9554 & +1.9144 & 0.26 & 0.37 & 0.27 \\
\hline 119 & SXS:BBH:0274 & 22.4 & 0.19 & $(3,-0.23,+0.85)$ & -1.0771 & +0.2083 & +0.2309 & +0.2277 & 0.13 & 0.13 & 0.13 \\
\hline 120 & SXS:BBH:0280 & 23.6 & 0.19 & $(3,+0.27,+0.85)$ & +0.6050 & +1.4186 & +0.8745 & +0.8733 & 0.28 & 0.32 & 0.31 \\
\hline 121 & SXS:BBH:0273 & 22.9 & 0.19 & $(3,-0.27,-0.85)$ & -1.3195 & +1.9565 & +1.6140 & +1.5855 & 0.12 & 0.14 & 0.09 \\
\hline 122 & SXS:BBH:0282 & & & $(3,+0.3$ & -0.0463 & +0.9797 & & +1.0064 & 0.06 & 9 & 0.09 \\
\hline 123 & SXS:BBH:0281 & 23.2 & 0.19 & $(3,+0.30,-0.30)$ & +0.0210 & +1.1088 & +1.2655 & +1.2626 & 0.10 & 0.18 & 0.18 \\
\hline 124 & SXS:BBH:0272 & 22.7 & 0.19 & $(3,-0.30,+0.30)$ & -0.0059 & +0.1127 & +0.1981 & +0.1941 & 0.04 & 0.03 & 0.03 \\
\hline 125 & SXS:BBH:0271 & 22.5 & 0.19 & $(3,-0.30,+0.00)$ & +0.0003 & +0.4184 & +0.4446 & +0.4403 & 0.03 & .03 & 0.03 \\
\hline 126 & SXS:BBH:0285 & 23.8 & 0.19 & $(3,+0.40,+0.60)$ & +1.2523 & +0.9367 & +0.5573 & +0.5566 & 0.12 & 0.14 & 0.14 \\
\hline 127 & SXS:BBH:0284 & 8 & 0.19 & $(3,+0.40,-0.60)$ & -0.4849 & +1.1027 & +1.4184 & +1.4149 & 0.29 & 0.26 & 0.26 \\
\hline 128 & SXS:BBH:0269 & 3 & 0.19 & $(3,-0.40,+0.60)$ & -0.9915 & -0.3676 & -0.2150 & -0.2181 & 0.07 & 0.05 & 0.05 \\
\hline 129 & SXS:BBH:0268 & 9 & 0 . & $(3,-0.40,-0.60)$ & -0.8497 & +1.2626 & +1.0233 & +1.0199 & 0.09 & 8 & 0.08 \\
\hline 130 & SXS:BBH:0174 & 5 & 0.19 & $(3,+0.50,+0.00)$ & -0.6660 & +1.2089 & +1.4297 & +1.4301 & 0.09 & 4 & 0.13 \\
\hline 131 & SXS:BBH:0045 & 0 & 0.19 & $(3,+0.50,-0.50)$ & & +0.0866 & +0.5459 & +0.4961 & 0.29 & 0.37 & 0.37 \\
\hline 132 & SXS:BBH:0290 & 24.2 & 0.19 & ,+0.40) & +0.1787 & -0.3282 & -0.5082 & -0.5447 & 0.11 & 0.17 & 0.18 \\
\hline 133 & SXS:BBH:0289 & 23.8 & 0.19 & $(3,+0.60,+0.00)$ & +1.8791 & -0.5607 & -0.3307 & -0.3699 & 0.09 & 0.08 & 0.06 \\
\hline 134 & SXS:BBH:0288 & 23.5 & 0.19 & $(3,+0.60,-0.40)$ & +1.9707 & -0.4652 & +0.0664 & +0.0241 & 0.30 & 0.40 & 0.40 \\
\hline 135 & SXS:BBH:0287 & 23.5 & 0.19 & $(3,+0.60,-0.60)$ & -0.2407 & -0.2999 & +0.3673 & +0.3176 & 0.45 & 0.61 & 0.61 \\
\hline 136 & SXS:BBH:0263 & 22.0 & 0.19 & $(3,-0.60,+0.60)$ & -0.9226 & -1.1034 & -0.8608 & -0.8634 & 0.18 & 0.12 & 0.12 \\
\hline 137 & SXS:BBH:0266 & 22.0 & 0.19 & $(3,-0.60,+0.40)$ & +1.3841 & -0.9093 & -0.7034 & -0.7060 & 0.13 & 0.09 & 0.09 \\
\hline 138 & SXS:BBH:0262 & 22.5 & 0.19 & $(3,-0.60,+0.00)$ & +1.2380 & -0.3905 & -0.2917 & -0.2943 & 0.05 & 0.04 & 0.04 \\
\hline 139 & SXS:BBH:0265 & 23.4 & 0.19 & $(3,-0.60,-0.40)$ & +2.1253 & +0.3689 & +0.3143 & +0.3114 & 0.06 & 0.05 & 0.05 \\
\hline 140 & SXS:BBH:0292 & 23.9 & 0.19 & $(3,+0.73,-0.85)$ & -0.6610 & -1.4035 & -0.4570 & -0.4977 & 1.05 & 1.45 & 0.18 \\
\hline 141 & SXS:BBH:0261 & 21.5 & 0.19 & $(3,-0.73,+0.85)$ & -0.6331 & -1.6990 & -1.3883 & -1.3902 & 0.33 & 0.22 & 0.22 \\
\hline 142 & SXS:BBH:0061 & 34.5 & 0.14 & $(5,+0.50,+0.00)$ & & +0.5422 & +0.8040 & +0.7322 & 0.03 & 0.04 & 0.05 \\
\hline 143 & SXS:BBH:0208 & 49.9 & 0.14 & $(5,-0.90,+0.00)$ & +8.8938 & +3.2712 & +3.6577 & +3.6624 & 0.08 & 0.08 & 0.08 \\
\hline 144 & SXS:BBH:0202 & 62.1 & 0.11 & $(7,+0.60,+0.00)$ & +1.2013 & -0.1358 & -0.1405 & -0.2271 & 0.15 & 0.15 & 0.18 \\
\hline 145 & SXS:BBH:0203 & 58.5 & 0.11 & $(7,+0.40,+0.00)$ & +8.0859 & +1.7976 & +1.7958 & +1.7585 & 0.02 & 0.02 & 0.03 \\
\hline 146 & SXS:BBH:0204 & 88.4 & 0.11 & $(7,+0.40,+0.00)$ & +16.4975 & +0.0118 & +0.0098 & -0.0472 & 0.09 & 0.09 & 0.09 \\
\hline 147 & SXS:BBH:0205 & 44.9 & 0.11 & $(7,-0.40,+0.00)$ & +4.4086 & +0.9674 & +0.9671 & +0.9641 & 0.03 & 0.03 & 0.03 \\
\hline 148 & SXS:BBH:0206 & 73.2 & 0.11 & $(7,-0.40,+0.00)$ & +11.0775 & -0.4651 & -0.4655 & -0.4959 & 0.11 & 0.11 & 0.11 \\
\hline 149 & SXS:BBH:0207 & 36.1 & 0.11 & $(7,-0.60,+0.00)$ & -1.3594 & +0.2969 & +0.2974 & +0.2680 & 0.03 & 0.06 & 0.06 \\
\hline
\end{tabular}

